\title{
Development of opioid-induced hyperalgesia depends on reactive astrocytes controlled by Wnt5a signaling
}

Short title: Astrogliosis in opioid-induced hyperalgesia

One sentence summary: Neuron-to-astrocyte Wnt5a signaling controls the pathogenesis of opioid-induced hyperalgesia via astrogliosis

\section{Xin Liu ${ }^{1,2}$, Chilman Bae ${ }^{1,3}$, Bolong Liu ${ }^{1,4}$, Yongmei Zhang ${ }^{1,5}$, Xiangfu Zhou ${ }^{4}$, Terry P. Yamaguchi ${ }^{6}$, Jin Mo Chung ${ }^{1}$, Shao-Jun Tang ${ }^{1,2, *}$}

${ }^{1}$ Department of Neuroscience and Cell Biology, University of Texas Medical Branch, Galveston, TX 77555, USA; '2Department of Anesthesiology, Renaissance School of Medicine, Stony Brook University, Stony Brook, NY 11794, USA; ${ }^{3}$ School of Electrical, Computer, and Biomedical Engineering, Southern Illinois University, Carbondale, IL 62901, USA; ${ }^{4}$ Department of Urology, The Third Affiliated Hospital of Sun Yat-Sen University, 600 W Tianhe Rd, Guangzhou, 510630, China; ${ }^{5}$ Jiangsu Province Key Laboratory of Anesthesiology, Xuzhou Medical University, Xuzhou, Jiangsu Province 221004, China; ${ }^{6}$ Center for Cancer Research, Cancer and Developmental Biology Laboratory, Cell Signaling in Vertebrate Development Section, NCl-Frederick, NIH, Frederick, MD 21702, USA. *Corresponding Author: shtang@utmb.edu 


\section{Abstract.}

Opioid analgesics are the frontline pain medicine for managing various types of pain. Paradoxically, repeated use of opioid analgesics may cause an exacerbated pain state known as opioid-induced hyperalgesia (OIH). OIH significantly contributes to dose escalation and consequently opioid overdose. In addition to neuronal malplasticity, emerging evidence suggests a critical role of reactive glia in OIH development. A potential astrocytic underpinning of OIH pathogenesis is indicated by their prominently activation in OlH animal models. However, this hypothesis has not been conclusively tested and the mechanism underlying the astrocyte activation remains unclear. Here, we show that reactive astrocytes (a.k.a. astrogliosis) are critical for OIH development in mice. Genetic ablation of astrogliosis inhibited the expression of OIH and morphine-induced neural circuit polarization (NCP) in the spinal dorsal horn (SDH). We also found that Wnt5a is a neuron-to-astrocyte signal that is required for morphine-induced astrogliosis. Conditional knock-out of Wnt5a in neurons or its co-receptor ROR2 in astrocytes blocked not only morphine-induced astrogliosis but also OIH and NCP. Furthermore, we showed that the Wnt5a-ROR2 signaling-dependent astrogliosis contributes to OlH via inflammasome-regulated IL-1 $\beta$. Our results reveal an important role of morphine-induced astrogliosis in OlH pathogenesis and elucidate a neuron-to-astrocyte intercellular Wnt signaling pathway that controls the astrogliosis.

\section{INTRODUCTION}

Opioid analgesics such as morphine are the gold standard for treating severe pain. However, among other side effects, chronic use or abuse of opioids paradoxically increases pain sensitivity, known as opioid-induced hyperalgesia $(\mathrm{OIH})^{1-4}$. OIH is a critical contributor to the common clinical practice of dose escalation for effective pain relief and opioid overdose $e^{4-6}$. Despite its clinical significance, the mechanisms of $\mathrm{OlH}$ pathogenesis are still poorly understood, hampering the development of effective interventions.

Several forms of malplasticity in pain neuronal circuits are implicated in $\mathrm{OIH}$ pathogenesis. These include opioid-induced sensitization of nociceptors in peripheral, sensitization of second order neurons in the spinal cord, and enhanced descending facilitation ${ }^{4,7}$. Long-term potential (LTP) of synapses between nociceptive $C$ fibers and second order neurons in the spinal cord dorsal horn is considered as a critical synaptic mechanism facilitating $\mathrm{OIH}$ expression ${ }^{7,8}$. Indeed, LTP and OIH appear to use similar molecular signaling pathways that involve NMDA receptors, CaMKII and BDNF to support their expression ${ }^{7}$. LTP might provide a synaptic support to enhance communication between pronociceptive neurons. It is less clear whether other forms of synaptic plasticity in the pain neuronal circuits contribute to $\mathrm{OlH}$ expression.

In addition to neuronal malplasticity, opioids also induce reactions of glia, 
including microglia and astrocytes ${ }^{7}$. Microglia were reported to play a critical role in $\mathrm{OIH}^{9}$ [but see also refs ${ }^{1,10}$. Astrocytes are activated in the spinal cords following different paradigms of opioid administration ${ }^{11,12}$. However, the potential role of reactive astrocytes in $\mathrm{OlH}$ expression has not been conclusively established, and the mechanism by which opioids cause the activation of astrocytes has not elucidated.

Astrocytes intimately interact with neurons, especially with synapses to form tripartite synaptic structures ${ }^{13}$. By interacting with synapses, astrocytes are positioned to sense and respond to synaptic activity-regulated signals from neurons. Because reactive astroglia are widely observed in pain pathways of various models of pathological pain ${ }^{14}$, it is tempting to conceive that pain-associated hyper-synaptic activity may modulate the activation of astrocytes. However, such neuron-to-astrocyte signals have not been elucidated, and their contribution to $\mathrm{OlH}$ expression has not been established.

On the other hand, astrocytes may also regulate neuronal functions. Under normal physiological conditions, astrocytes play essential roles in regulating central nervous system homeostasis ${ }^{15}$, plasticity of neural circuits $^{16}$, and synaptic transmission 13,17,18. However, how reactive astrocytes may regulate synaptic transmission and plasticity remains elusive. In particular, it is unclear whether reactive astrocytes contribute to malsynaptic plasticity of pain circuits associated with $\mathrm{OIH}$ development. Although reactive astrocytes are thought to dysregulate neuronal circuits by secreting bioactive molecules such as chemokines and cytokines, the involvement of such a mechanism in $\mathrm{OIH}$ pathogenesis has not been tested directly.

In this study, we use a mouse $\mathrm{OlH}$ model to show that reactive astrocytes are critical for morphine-induced $\mathrm{OIH}$ and malsynaptic plasticity in the $\mathrm{SDH}$. We also demonstrate that the morphine-elicited astrocyte activation is controlled by a neuron-to-astrocyte intercellular Wnt5a-ROR2 signaling pathway. We further elucidate that the reactive astrocytes promote $\mathrm{OIH}$ and malsynaptic plasticity via inflammasome-regulated IL-1 $\beta$.

\section{RESULTS}

\section{Reactive astrocytes play essential role in OIH expression and maintenance.}

To induce OlH, we repeatedly injected wild-type (WT; C57BL) mice with morphine (i.p.; $20 \mathrm{mg} / \mathrm{kg} / \mathrm{day}$ for 4 days). We observed the expression of mechanical $\mathrm{OIH}$ following the morphine administration (Fig. 1C), as reported previously ${ }^{2,19}$. In these animal models, we found microglia and astrocyte activation in the spinal dorsal horn $(\mathrm{SDH})$ (Fig. 1A-1B), consistent with previous observations ${ }^{20,21}$. A critical role of microglia in $\mathrm{OIH}$ was proposed in an early study ${ }^{9}$, but inconsistent findings were reported later ${ }^{1,10}$. In this study, we aimed to examine the potential role of reactive astrocytes in OIH development. To this end, we employed a genetic approach to selectively ablate reactive astrocytes, using GFAP-thymidine kinase (TK) transgenic mice $^{22}$. The transgenic mice were administered with ganciclovir (GCV), which would be metabolized to nucleotide analogues by TK in astrocytes. The GCV-derived nucleotide analogues are toxic to proliferating cells, and thus would specifically induce cell death of reactive astrocytes. 
Von Frey tests showed that the GFAP-TK transgenic mice expressed mechanical OIH similarly as WT mice (Fig. 1C). To determine the contribution of astrogliosis to the expression of $\mathrm{OIH}$, we intrathecally (i.t.) injected GCV $(5 \mathrm{mg} / \mathrm{kg})$ during the first 2 days of morphine administration. We observed that GCV administration blocked the morphine-induced increase of GFAP but not lba1 (Fig. 1A-1B), indicating selective ablation of reactive astrocytes without affecting microglia. Importantly, the GCV treatment impaired OlH expression in GFAP-TK but not in WT mice (Fig. 1C). Significant GCV-induced impairment of OIH in the transgenic mice started to develop one day after the first GCV injection, suggesting a critical role of reactive astrocytes in early expression of $\mathrm{OlH}$.

We then sought to determine if astrogliosis contributes to OIH maintenance. To this end, we tested the effect of ablation of reactive astrocytes on established $\mathrm{OIH}$. In this experiment, GCV was administered six days after the first injection of morphine, when OIH was fully expressed (Fig. 1D). We observed that the GCV administration significantly reversed the established OIH in GFAP-TK but not in WT mice (Fig. 1D), indicating an important role of reactive astrocytes in $\mathrm{OlH}$ maintenance. These results collectively showed that reactive astrocytes were essential for both expression and maintenance of $\mathrm{OlH}$.

\section{Reactive astrocytes mediate morphine-induced neural circuit polarization in the SDH.}

To gain insight into the neural circuitry mechanism by which astrogliosis contributes to $\mathrm{OIH}$, we tested the effect of astrogliosis ablation on morphine-induced malplasticity in pain neural circuits. When we performed whole-cell recording of SDH neurons in morphine-treated GFAP-TK mice, we found that morphine increased both the frequency of spontaneous excitatory postsynaptic currents (sEPSCs) and the amplitude of evoked EPSCs (eEPSCs) of excitatory neurons (Fig. 2A-2C), identified by their characteristic non-tonic firing patterns ${ }^{23,24}$. By contrast, morphine decreased the sEPSC frequency and eEPSC amplitude of inhibitory neurons (Fig. 2D-2F), identified by their characteristic tonic firing pattern ${ }^{23,24}$. These findings indicate that morphine administration polarizes neural circuits in the $\mathrm{SDH}$, by increasing excitatory inputs to excitatory neurons and meanwhile decreasing excitatory inputs to inhibitory neurons. The observed neural circuit polarization (NCP) might contribute to $\mathrm{OIH}$ expression, by facilitating activation of the SDH pain pathway.

Astrocytes normally play important role in maintaining homeostasis of neural circuits ${ }^{15}$, but it is unclear how astrogliosis modulates neural circuits during pain pathogenesis. Hence, we set to test the contribution of astrogliosis to morphine-induced NCP. To this end, we determined the effect of astrogliosis ablation on EPSCs of excitatory and inhibitory neurons in the SDH of GFAP-TK transgenic mice. We found that GCV treatment abolished the morphine-induced increase in sEPSC frequency and eEPSC amplitude of excitatory neurons (Fig. 2A-2C).

On inhibitory neurons, we observed that GCV treatment by itself decreased sEPSC frequency and eEPSC amplitude of inhibitory neurons in GFAP-TK mice without morphine administration (Fig. 2D-2F), indicating excitatory inputs on inhibitory 
neurons under physiological conditions are more sensitive to astrocyte ablation than excitatory inputs on excitatory neurons. Importantly, after GCV treatment morphine failed to induce further decease of SEPSC frequency and eEPSC amplitude on inhibitory neurons (Fig. 2D-2F). Together, these results indicated that astrogliosis was critical for morphine to induce NCP, including the increase of SEPSC frequency and eEPSC amplitude on excitatory neurons and the decrease of sEPSC frequency and eEPSC amplitude on inhibitory neurons.

\section{Neuronal Wnt5a is critical for morphine to induce astrogliosis, NCP and OIH.}

Having shown the essential role of astrogliosis in OIH and NCP, we next sought to elucidate the mechanism by which morphine induces astrocyte activation. To this end, we focused on the potential role of Wnt5a signaling, which is implicated in pain pathogenesis ${ }^{25-27}$. Wnt5a is secreted protein mainly expressed by neurons ${ }^{28}$, and is upregulated by in the SDH by pain signals ${ }^{29}$. Wnt5a secretion is controlled by synaptic activity $^{30,31}$. The involvement of Wnt5a in regulating astrogliosis is suggested by the observations that Wnt5a antagonist inhibits astrocyte activation in pain models ${ }^{32,33}$ and JNK, a critical downstream target in the Wnt5a signaling pathway, is critical for astrogliosis in a neuropathic pain model ${ }^{34}$. Based on these prior findings, we hypothesized that Wnt5a secreted from neurons hyperactivated following morphine treatment activated astrocytes. To test this hypothesis, we generated neuronal Wnt5a conditional knock-out (CKO) mice (Wnt5a-CKO-S) by crossing floxed Wnt5a mice ${ }^{35}$ with synapsin 1-Cre mice ${ }^{36}$. We observed that morphine administration significantly upregulated spinal Wnt5a protein in the WT but not in Wnt5a-CKO-S mice (Fig. 3A), indicating morphine stimulated Wnt5a upregulation predominantly in spinal neurons. Importantly, we found that, unlike WT mice, morphine completely failed to induce GFAP upregulation in the spinal cord of the Wnt5a-CKO-S mice (Fig. 3B), indicating deletion of Wnt5a in neurons abolished morphine-induced astrogliosis. To confirm these findings, we also generated another Wnt5a CKO mutant to delete Wnt5a in neural stem cells using nestin-Cre ${ }^{37}$ (Wnt5a CKO-N). We found that this Wnt5a mutation also abolished morphine-induced Wnt5a upregulation and astrogliosis (Sup. Fig. 1). These findings collectively showed that neuronal Wnt5a was essential for morphine to induce astrogliosis.

As reactive astrocytes were crucial for OIH induced by morphine (Fig. 1C, 1D) and neuronal Wn5a was required for the astrocyte activation (Fig. 3B), we hypothesized that the $\mathrm{OIH}$ expression depended on neuronal Wnt5a. To test this hypothesis, we determined the effect of neuronal Wnt5a CKO on $\mathrm{OlH}$. The result showed that OlH expression was abolished in Wnt5a CKO-S mice (Fig. 3C). We observed similar inhibitory effect on OIH in Wnt5a CKO-N mice (Fig. 3D). These data together demonstrated the requirement of neuronal Wnt5a for OIH development.

Because of the observed role of astrogliosis in NCP (Fig. 2) and neuronal Wnt5a in the astrogliosis (Fig. 3B), we further hypothesized that neuronal Wnt5a was important for NCP expression. To test this, we determined the expression of NCP in the SDH of Wnt5a CKO-S mice. We found that the morphine-induced increase of sEPSC frequency of excitatory neurons (Fig. 3E) and the decrease of sEPSC 
frequency of inhibitory neurons (Fig. 3F) were abolished in Wnt5a CKO-S mice. These results showed that neuronal Wnt5a mediated the expression of NCP in the $\mathrm{SDH}$.

\section{Astrocytic ROR2 receptor is crucial for astrogliosis, NCP and OIH induced by morphine.}

The results described above demonstrate a key role of Wnt5a from neurons in mediating morphine-induced astrocyte activation. Next, we sought to further elucidate the underlying mechanism by which Wnt5a activates astrocytes. We hypothesized that Wnt5a co-receptor ROR2 on astrocytes ${ }^{28}$ was critical for the astrogliosis. To test this hypothesis, we generated astrocytic CKO of ROR2 by crossing floxed ROR2 ${ }^{38}$ and GFAP-Cre ${ }^{39}$ mouse lines. Similar to the effect of neuronal Wnt5a CKO (Fig. 3), we observed that the astrocytic ROR2 CKO also blocked morphine-induced GFAP upregulation, indicating inhibition of astrogliosis (Fig. 4A).

As astrogliosis and neuronal Wnt5a are critical for OIH development (Fig. 1; Fig. 3 ), the finding of the astrogliosis blockage by the ROR2 CKO led us to predict that the mutant mice would be impaired in OlH development. In support of this hypothesis, we indeed found that the expression of OlH was abolished in the ROR2 CKO mice (Fig. 4B), as in the Wnt5a CKO-S and Wnt5a CKO-N mice (Fig. 3).

Because of the observed contribution of astrogliosis and neuronal Wnt5a to NCP (Fig. 2; Fig. 3), we further hypothesized that the ROR2 CKO would impair the expression of NCP. We tested this hypothesis by determining effect of the ROR2 CKO on sEPSCs of neurons in the SDH. The results showed that both the morphine-induced increase in SEPSC frequency of excitatory neurons and decrease in SEPSC frequency of inhibitory neurons in the SDH were blocked in ROR2 CKO mice (Fig. 4C-4D). The findings suggested that, similar to neuronal Wnt5a, astrocytic ROR2 also played an important role in morphine-induced astrogliosis, OIH, and NCP.

\section{Wnt5a signaling-regulated reactive astrocytes control OIH and NCP via IL-1 $\beta$.}

The above results from Wnt5a CKO and ROR2 CKO mice collectively suggest that Wnt5a secreted from neurons stimulates astrocytic ROR2 receptor to promote morphine-induced astrogliosis, and that this neuron-to-astrocyte Wnt5a-ROR2 signaling pathway contributes to $\mathrm{OlH}$ expression via astrogliosis. Next, we sought to further understand the mechanism by which Wnt5a signaling-mediated astrogliosis regulates $\mathrm{OIH}$ pathogenesis. Previous studies reveal that reactive astrocytes contribute to pain pathogenesis by releasing proinflammatory mediators ${ }^{14}$. Indeed, we observed morphine-induced upregulation of spinal activated (cleaved) IL-1 $\beta$ (Fig. 5A), a key proinflammatory cytokine that is implicated in $\mathrm{OlH}^{40}$ and regulated by Wnt5a signaling $^{28}$. Importantly, CKO of Wnt5a in either Wnt5a CKO-S or CKO-N mice abolished the IL-1 $\beta$ upregulation (Fig. 5A). CKO of ROR2 in astrocytes also blocked IL-1 $\beta$ upregulation (Fig. 5A). These results suggested that the neuron-to-astrocyte Wnt5a-ROR2 signaling controlled morphine-induced IL-1 $\beta$ upregulation.

Because the neuron-to-astrocyte Wnt5a-ROR2 signaling controls both astrocyte activation (Fig. 3; Fig.4) and IL-1 $\beta$ upregulation induced by morphine (Fig. 5A), we 
hypothesized that the reactive astrocytes produced IL-1 $\beta$. To test this hypothesis, we determined the effect of ablating reactive astrocytes on the $\mathrm{IL}-1 \beta$ upregulation. We found that astrogliosis ablation by GCV in GFAP-TK transgenic mice significantly impaired morphine-induced IL-1 $\beta$ upregulation in the spinal cord (Fig. 5B). On the other hand, ablation of microglia by the CSF1R inhibitor PLX5622 $2^{41-43}$ did not significantly affect morphine-induced spinal IL-1 $\beta$ upregulation (Fig. 5C). These results suggested that reactive astrocytes, rather than microglia, were the major cell source of morphine-induced IL-1 $1 \beta$. Previous studies suggest astrocytic IL-1 $\beta$ in other pain models ${ }^{11}$.

Because reactive astrocytes contributed to morphine-induced $\mathrm{OlH}$ (Fig. 1) and produced IL1 $\beta$ (Fig. 5B), we hypothesized that astrogliosis promoted OIH via IL-1 $\beta$. Hence, we tested the pathogenic role of $\mathrm{IL}-1 \beta$ in $\mathrm{OlH}$ expression. In these experiments, we used the endogenous IL-1 receptor antagonist IL-1Ra to block II-1 $\beta$ signaling ${ }^{40}$. We observed that IL-1Ra administration $(20 \mu \mathrm{g} / \mathrm{kg} /$ day for the first 3 days, i.t.) abolished the expression of $\mathrm{OlH}$, without affecting baseline mechanical sensitivity (Fig. 6A). These results suggested that IL-1 $\beta$ produced by reactive astrocytes was critical for $\mathrm{OIH}$ pathogenesis.

IL-1Ra administration also blocked the morphine-induced increase in sEPSC frequency (Fig. 6B) and eEPSC amplitude (Fig. 6C-6D) in excitatory neurons in the $\mathrm{SDH}$. Furthermore, using GAD67-GFP transgenic mice ${ }^{44}$, we found that IL-1Ra blocked the morphine-induced decrease in sEPSC frequency (Fig. 6E) and sEPSC amplitude (Fig. 6F-6G) of GABAergic inhibitory neurons in the SDH. Together, these results indicated that the morphine-induced expression of NCP was controlled by IL-1 $\beta$ produced by Wnt5a-ROR2 signaling-activated astrocytes (Fig. 5A-5B).

\section{Wn5a-ROR2 signaling activates IL-1 $\beta$ via Inflammasomes.}

Having identified a critical of IL-1 $\beta$ in $\mathrm{OIH}$, we wanted to further understand the mechanism by which morphine regulates astrocytic IL-1 $\beta$. We hypothesized inflammasome, a key protein complex that controls $\mathrm{IL}-1 \beta$ processing ${ }^{45}$ and is regulated by morphine to prolong neuropathic pain ${ }^{46}$, played a key role in this process. We observed that morphine administration upregulated the active forms of both IL-1 $\beta$ and caspase 1 (Cas-1), the inflammasome proteinase that processes pro-IL-1 $\beta$ protein (Fig. 7A). In addition, pharmacological blockade of the inflammasome with AC-YVAD-CMK, a selective inhibitor of Cas-1, impaired the upregulation of Cas- 1 and $\mathrm{IL}-1 \beta$ (Fig. 7A). These results indicated that morphine stimulated spinal IL- $\beta$ activation via the inflammasome. Furthermore, inhibition of the inflammasome by eAC-YVDA-CMK or Cas-1 siRNA also blocked the expression of OIH (Fig. 7B-7C). These data collectively demonstrated a critical role of inflammasome in the regulation of IL-1 $\beta$ during $\mathrm{OIH}$ expression.

Because morphine induced IL-1 $\beta$ via Wnt5-ROR2 signaling-regulated astrogliosis (Fig. 3; Fig. 4; Fig. 5B), we hypothesized the neuron-to-astrocyte Wnt5a-ROR2 signaling regulated morphine-induced inflammasome activation. To test this idea, we determined the effect of neuronal Wnt5a CKO and astrocytic ROR2 CKO on inflammasome activation, by measuring active Cas- 1 levels. We found that either the 
Wnt5a CKO-S or ROR2 CKO abolished the morphine-induced increase of active Cas-1 (Fig. 7D). These findings suggested that Wnt5a-ROR2 signaling regulated the activation of inflammasome to process IL-1 $\beta$ in astrocytes.

\section{DISCUSSION}

We show that astrogliosis is crucial for morphine-induced $\mathrm{OlH}$ and expression of $\mathrm{NCP}$ in the SDH. The morphine-induced astrogliosis, NCP, and OIH requires Wnt5a in neurons and the ROR2 receptor in astrocytes. Furthermore, the effects of morphine on neural circuits and pain behavior are mediated by IL- $\beta$ controlled by Wnt5a-ROR2 pathway-regulated inflammasome activation. These findings suggest bidirectional interactions between neurons and astrocytes during the pathogenesis of $\mathrm{OlH}$. We propose that morphine induces Wnt5a release from neurons to stimulate astrocytic ROR2 receptor to activate astrocytes and inflammasome to produce $\mathrm{IL}-1 \beta$, and active $\mathrm{IL}-1 \beta$ released from reactive astrocytes in return stimulates pain processing neurons in the SDH to promote NCP and OIH (Fig. 8).

\section{Neuronal circuitry mechanism in the development of OlH.}

Malplasticity of multiple components in the pain transmission pathway is implicated in OIH. Sensitization of peripheral nociceptors and second-order neurons in the spinal cord, long-term potentiation (LTP), and descending facilitation are among the best characterized forms of neuronal malplasticity proposed for $\mathrm{OlH}$ expression $3,5,7,8,47,48$. These and other lines of evidence suggest the importance of malplasticity of excitatory systems in OlH expression ${ }^{7}$. However, because functional homeostasis of normal neural circuits is maintained by the coordinated activity of excitatory and inhibitory neurons, it is also important to understand if and how this coordination is altered during $\mathrm{OlH}$ development. Yet, we currently know little in this aspect. We find that morphine indeed induces hyper-activity of excitatory inputs on excitatory neurons and hypo-activity of excitatory inputs on inhibitory neurons (Fig. 2). The increased synaptic drive on excitatory neurons and decreased synaptic drive on inhibitory neurons reveal a form of morphine-induced polarization in the SDH neural circuit. This form of neural circuit polarization (NCP) would facilitate hyper-activation of the pain neural circuit in the SDH and thus promote the expression of $\mathrm{OIH}$. This neural circuitry mechanism may also play a critical role in HIV-1 gp120-induced pain pathogenesis ${ }^{49}$.

The increase of excitatory synaptic drive on excitatory neurons is consistent with the involvement of sensitization of pain processing neurons in the $\mathrm{SDH}$ during $\mathrm{OIH}$ expression, as suggested by previous studies ${ }^{7}$. It is unexpected for us to observe the decrease of excitatory synaptic drive on inhibitory neurons. Inhibitory loss, manifested as loss of inhibitory neurons or synapses, has been suggested previously as a potential mechanism underlying pain pathogenesis ${ }^{50-52}$. Our findings reveal novel form of loss of inhibition implicated in OlH development.

Our finding of NCP reveals a novel circuit mechanism that supports the expression of $\mathrm{OlH}$. The increase excitatory inputs to excitatory neurons and the decrease of excitatory inputs to inhibitory neurons in NCP may promote $\mathrm{OlH}$ by facilitating hyper-activation of pain transmission circuits. 


\section{Astrocytic mechanism in regulation of OlH expression.}

Previous studies on $\mathrm{OlH}$ mechanism mainly concentrate on the contribution of neuronal plasticity. The involvement of glial cells is recognized later, as indicated by the activation of microglia and astrocytes in rodent $\mathrm{OlH}$ models ${ }^{7}$. Microglia were suggested to modulate neuronal activity via BDNF during OIH expression ${ }^{9}$. However, other studies raise questions about the role of microglia ${ }^{1,10}$. Astrogliosis is associated with various chronic pain conditions, including $\mathrm{OlH}$ models, but its pathogenic contribution has not been tested conclusively ${ }^{7,14}$. Previous work suggests a contribution of activated astrocytes to pain development in animal models ${ }^{53,54}$. However, a recent study by Sasaki et al. show that inhibition of astrocyte activation by gap junction blockade did not ameliorate $\mathrm{OIH}^{55}$. It is important to note that the GFAP upregulation induced by morphine was not blocked in this study, raising uncertainty about the degree of astrogliosis inhibition.

Our current speculations of astrocytic function in pain pathogenesis are still largely based on circumventive evidence such as the correlation between astrogliosis and pain and the effect of glial inhibitors ${ }^{53-55}$. We take a genetic approach to specific ablate reactive astrocytes and thus directly evaluate the contribution of reactive astrocytes to $\mathrm{OIH}$. We show that astrogliosis ablation during the early phase of $\mathrm{OIH}$ induction impaired the expression of mechanical OIH (Fig. 1C), whereas astrogliosis ablation after the establishment of $\mathrm{OIH}$ significantly reversed the OIH (Fig. 1D). These results strongly suggest an important role of reactive astrocytes in both expression and maintenance of $\mathrm{OlH}$.

Our conclusion described above is also supported by the effect of astrogliosis inhibition induced by the CKO of Wnt5a or ROR2. We show that when morphine-induced astrogliosis is blocked by either neuronal Wnt5a CKO or astrocytic ROR2 CKO OIH is abolished (Fig. 3; Fig. 4). These cell-type-specific approaches of astrogliosis inhibition provide strong complementary evidence for a crucial contribution of reactive astrocytes to $\mathrm{OlH}$.

How would reactive astrocytes promote $\mathrm{OlH}$ ? Little is known about the answer to this important question. Astrocytic processes intimately interact with synapses to form a tripartite synaptic structure ${ }^{13}$. Under physiological conditions, normal astrocytes play key roles in neural circuit homeostasis, by maintaining cation (e.g. $\mathrm{K}_{+}$) equilibrium, uptaking released neurotransmitters and releasing gliotransmitters ${ }^{56}$. However, it is unclear how reactive astrocytes would disturb the circuit homeostasis, especially in during $\mathrm{OlH}$ development. We find that morphine induces NCP and ablation of astrogliosis suppresses the NCP expression (Fig. 2). These findings suggest that morphine-induced reactive astrocytes disturb circuit homeostasis and mediate the expression of NCP. This notion is supported by the findings that inhibition of astrogliosis in either the neuronal Wnt5a CKO or the astrocytic ROR2 CKO mouse also blocks NCP expression (Fig. 3; Fig. 4). Based on these results, we propose that reactive astrocytes facilitates $\mathrm{OIH}$ by disrupting homeostasis of neural circuits in the SDH and promoting NCP expression.

Then, how would reactive astrocytes promote NCP? Based on the characterized 
biological activity of reactive astrocytes, it has been speculated that they could facilitate $\mathrm{OlH}$ expression by releasing excitatory substances to stimulate synapses. The suggested excitatory factors include proinflammatory cytokines and chemokines, ATP and gliotransmitters ${ }^{7}$. However, this hypothesis has not been directly and systematically tested in the context of $\mathrm{OlH}$. Our results suggest that the reactive astrocytes in the OIH models generate active IL-1 $\beta$ (Fig. 5). We further show that blockade of IL-1 signaling by IL-1Ra impairs both OIH and NCP (Fig. 6). Based on these findings, we suggest that reactive astrocytes contribute to NCP via IL-1 $\beta$.

\section{Wnt signaling in regulation of astrogliosis during OlH pathogenesis.}

Mechanism of astrocyte activation during $\mathrm{OlH}$ pathogenesis remains poorly understood. We find that neuronal Wnt5a and astrocytic ROR2 receptors are critical for morphine to activate astrocytes in the spinal cord (Fig. 3; Fig. 4). These findings suggest that Wnt5a secreted from neurons stimulates its receptor ROR2 on astrocytes to activate astrocytes. Our results also show that this neuron-to-astrocyte Wnt5a-ROR2 signaling pathway is required for OIH and NCP (Fig. 3; Fig. 4), both of which are dependent on reactive astrocytes (Fig.1; Fig. 2).

Wnt5a expression and secretion are evoked by synaptic activity ${ }^{30}$ and pain ${ }^{29}$. In addition, morphine is known to upregulate Wn5a in the spinal cord ${ }^{32}$. Hence, we propose that $\mathrm{OlH}$ induced by morphine elicits Wnt5a release from neurons to activate astrocytes. The requirement of neuronal Wnt5a indicates that morphine does not cause astrocyte activation by directly stimulating astrocytes in vivo. Instead, it activate astrocytes indirectly via neuronal Wnt5a. Consistent with this notion, $\mu$-opioid receptor (MOR), which is critical for $\mathrm{OlH}$ development and critical for astrocyte activation induced by ultra-low-dose morphine ${ }^{12}$, is not expressed in spinal astrocytes ${ }^{57}$.

Our data further show that Wnt5a receptor ROR2 on astrocytes is critical for morphine to activate astrocytes (Fig. 4). Because JNK signaling is an important intracellular mediator of the Wnt5a-ROR2 pathway ${ }^{58}$ and the JNK signaling are critical for astrocyte activation induced in $\mathrm{OIH}$ and neuropathic pain models ${ }^{12,34}$, it is likely that morphine induced Wnt5a-ROR2 signaling activate astrocytes via JNKs.

The neuron-to-astrocyte Wnt5a-ROR2 intercellular signaling pathway is also critical for spinal astrocyte activation induced by gp120 in an HIV pain model ${ }^{49}$, indicating that this pathway is a general mechanism that controls astrocyte activation induced by different neurological conditions.

Collectively, our results suggest that astrogliosis is a key cell target for $\mathrm{OlH}$ intervention. Furthermore, the results also suggest that the Wnt5a-ROR2 signaling pathway is a potential molecular target to block the astrogliosis during $\mathrm{OlH}$ pathogenesis.

\section{References}

1. Corder, G., et al. Loss of $\mu$ opioid receptor signaling in nociceptors, but not microglia, abrogates morphine tolerance without disrupting analgesia. Nature Medicine 23, 164 (2017).

2. Mao, J., Price, D.D. \& Mayer, D.J. Thermal hyperalgesia in association with 
the development of morphine tolerance in rats: roles of excitatory amino acid receptors and protein kinase C. J Neurosci 14, 2301-2312 (1994).

3. Angst, Martin S. \& Clark, J D. Opioid-induced Hyperalgesia: A Qualitative Systematic Review. Anesthesiology 104, 570-587 (2006).

4. Chu, L.F., Angst, M.S. \& Clark, D. Opioid-induced Hyperalgesia in Humans: Molecular Mechanisms and Clinical Considerations. The Clinical Journal of Pain 24, 479-496 410.1097/AJP.1090b1013e31816b31812f31843 (2008).

5. Servick, K. Primed for pain. Science 354, 569-571 (2016).

6. Miller, G. Pot and pain. Science 354, 566-568 (2016).

7. Roeckel, L.-A., Le Coz, G.-M., Gavériaux-Ruff, C. \& Simonin, F. Opioid-induced hyperalgesia: Cellular and molecular mechanisms. Neuroscience 338, 160-182 (2016).

8. Drdla, R., Gassner, M., Gingl, E. \& Sandkühler, J. Induction of Synaptic Long-Term Potentiation After Opioid Withdrawal. Science 325, 207-210 (2009).

9. Ferrini, F., et al. Morphine hyperalgesia gated through microglia-mediated disruption of neuronal Cl- homeostasis. Nature Neuroscience 16, 183 (2013).

10. Liu, X., Liu, B.-L., Yang, Q., Zhou, X. \& Tang, S.-J. Microglial ablation does not affect opioid-induced hyperalgesia in rodents. bioRxiv, 2021.2004.2027.441650 (2021).

11. Berta, T., Liu, Y.C., Xu, Z.Z. \& Ji, R.R. Tissue plasminogen activator contributes to morphine tolerance and induces mechanical allodynia via astrocytic IL-1 $\beta$ and ERK signaling in the spinal cord of mice. Neuroscience 247, 376-385 (2013).

12. Sanna, M.D., Ghelardini, C. \& Galeotti, N. Activation of JNK pathway in spinal astrocytes contributes to acute ultra-low-dose morphine thermal hyperalgesia. Pain 156, 1265-1275 (2015).

13. Perea, G., Navarrete, M. \& Araque, A. Tripartite synapses: astrocytes process and control synaptic information. Trends in neurosciences 32, 421-431 (2009).

14. Ji, R.-R., Donnelly, C.R. \& Nedergaard, M. Astrocytes in chronic pain and itch. Nature reviews. Neuroscience 20, 667-685 (2019).

15. Barres, B.A. The mystery and magic of glia: a perspective on their roles in health and disease. Neuron 60, 430-440 (2008).

16. Khakh, B.S. \& Sofroniew, M.V. Diversity of astrocyte functions and phenotypes in neural circuits. Nature neuroscience 18, $942-952$ (2015).

17. Allen, N.J. \& Eroglu, C. Cell Biology of Astrocyte-Synapse Interactions. Neuron 96, 697-708 (2017).

18. Lines, J., Martin, E.D., Kofuji, P., Aguilar, J. \& Araque, A. Astrocytes modulate sensory-evoked neuronal network activity. Nat Commun 11, 3689 (2020).

19. Li, X., Angst, M.S. \& Clark, J.D. A murine model of opioid-induced hyperalgesia. Brain research. Molecular brain research 86, 56-62 (2001).

20. Song, P. \& Zhao, Z.Q. The involvement of glial cells in the development of morphine tolerance. Neuroscience research 39, 281-286 (2001).

21. Hutchinson, M.R., et al. Possible involvement of toll-like receptor 4 /myeloid 
differentiation factor-2 activity of opioid inactive isomers causes spinal proinflammation and related behavioral consequences. Neuroscience 167, 880-893 (2010).

22. Bush, T.G., et al. Leukocyte infiltration, neuronal degeneration, and neurite outgrowth after ablation of scar-forming, reactive astrocytes in adult transgenic mice. Neuron 23, 297-308 (1999).

23. Punnakkal, P., von Schoultz, C., Haenraets, K., Wildner, H. \& Zeilhofer, H.U. Morphological, biophysical and synaptic properties of glutamatergic neurons of the mouse spinal dorsal horn. The Journal of Physiology 592, 759-776 (2014).

24. Todd, A.J. Identifying functional populations among the interneurons in laminae I-III of the spinal dorsal horn. Molecular Pain 13, 1744806917693003 (2017).

25. Yuan, S., Shi, Y. \& Tang, S.J. Wnt Signaling in the Pathogenesis of Multiple Sclerosis-Associated Chronic Pain. J Neuroimmune Pharmacology [Epub ahead of print] DOI: 10.1007/s11481-012-9370-3(2012).

26. Yuan, S., et al. A Wnt5a signaling pathway in the pathogenesis of HIV-1 gp120-induced pain. Pain 156, 1311-1319 (2015).

27. Simonetti, M. \& Kuner, R. Spinal Wnt5a Plays a Key Role in Spinal Dendritic Spine Remodeling in Neuropathic and Inflammatory Pain Models and in the Proalgesic Effects of Peripheral Wnt3a. J Neurosci 40, 6664-6677 (2020).

28. Li, B., et al. Wingless-type Mammary Tumor Virus Integration Site Family, Member 5A (Wnt5a) Regulates Human Immunodeficiency Virus Type 1 (HIV-1) Envelope Glycoprotein 120 (gp120)-induced Expression of Pro-Inflammatory Cytokines via the $\mathrm{Ca2}+/$ Calmodulin-dependent Protein Kinase II (CaMKII) and C-Jun N-terminal Kinase (JNK) Signaling Pathways. Journal of Biological Chemistry 288, 13610-13619 (2013).

29. Shi, Y., et al. Regulation of Wnt signaling by nociceptive input in animal models. Molecular Pain 8, 47 (2012).

30. Li, Y., et al. NMDA receptor activation stimulates transcription-independent rapid wnt5a protein synthesis via the MAPK signaling pathway. Molecular Brain 5, 1 (2012).

31. Chen, J., Park, C.S. \& Tang, S.J. Activity-dependent synaptic Wnt release regulates hippocampal long term potentiation. J Biol Chem 281, 11910-11916 (2006).

32. Shi, Y., Yuan, S. \& Tang, S.-J. Morphine and HIV-1 gp120 cooperatively promote pathogenesis in the spinal pain neural circuit. Molecular Pain 15, 1744806919868380 (2019).

33. Yuan, S., Shi, Y., Guo, K. \& Tang, S.-J. Nucleoside Reverse Transcriptase Inhibitors (NRTIs) Induce Pathological Pain through Wnt5a-Mediated Neuroinflammation in Aging Mice. Journal of Neuroimmune Pharmacology (2018).

34. Zhuang, Z.-Y., et al. A Peptide c-Jun N-Terminal Kinase (JNK) Inhibitor Blocks Mechanical Allodynia after Spinal Nerve Ligation: Respective Roles of JNK 
Activation in Primary Sensory Neurons and Spinal Astrocytes for Neuropathic Pain Development and Maintenance. The Journal of Neuroscience 26, 3551-3560 (2006).

35. Miyoshi, H., Ajima, R., Luo, C.T., Yamaguchi, T.P. \& Stappenbeck, T.S. Wnt5a potentiates TGF- $\beta$ signaling to promote colonic crypt regeneration after tissue injury. Science 338, 108-113 (2012).

36. Zhu, Y., et al. Ablation of NF1 function in neurons induces abnormal development of cerebral cortex and reactive gliosis in the brain. Genes \& Development 15, 859-876 (2001).

37. Tronche, F., et al. Disruption of the glucocorticoid receptor gene in the nervous system results in reduced anxiety. Nat Genet 23, 99 (1999).

38. Ho, H.-Y.H., et al. Wnt5a-Ror-Dishevelled signaling constitutes a core developmental pathway that controls tissue morphogenesis. Proceedings of the National Academy of Sciences 109, 4044-4051 (2012).

39. Garcia, A.D.R., Doan, N.B., Imura, T., Bush, T.G. \& Sofroniew, M.V. GFAP-expressing progenitors are the principal source of constitutive neurogenesis in adult mouse forebrain. Nat Neurosci 7, 1233 (2004).

40. Johnston, I.N., et al. A role for proinflammatory cytokines and fractalkine in analgesia, tolerance, and subsequent pain facilitation induced by chronic intrathecal morphine. J Neurosci 24, 7353-7365 (2004).

41. Hilla, A.M., Diekmann, H. \& Fischer, D. Microglia Are Irrelevant for Neuronal Degeneration and Axon Regeneration after Acute Injury. The Journal of Neuroscience 37, 6113-6124 (2017).

42. Janova, $\mathrm{H}$., et al. Microglia ablation alleviates myelin-associated catatonic signs in mice. The Journal of Clinical Investigation 128(2017).

43. Reshef, R., et al. The role of microglia and their CX3CR1 signaling in adult neurogenesis in the olfactory bulb. eLife 6, e30809 (2017).

44. Oliva, A.A., Jiang, M., Lam, T., Smith, K.L. \& Swann, J.W. Novel Hippocampal Interneuronal Subtypes Identified Using Transgenic Mice That Express Green Fluorescent Protein in GABAergic Interneurons. The Journal of Neuroscience 20, 3354-3368 (2000).

45. Schroder, K. \& Tschopp, J. The Inflammasomes. Cell 140, 821-832 (2010).

46. Grace, P.M., et al. Morphine paradoxically prolongs neuropathic pain in rats by amplifying spinal NLRP3 inflammasome activation. Proceedings of the National Academy of Sciences 113, E3441-E3450 (2016).

47. Araldi, D., Khomula, E.V., Ferrari, L.F. \& Levine, J.D. Fentanyl Induces Rapid Onset Hyperalgesic Priming: Type I at Peripheral and Type II at Central Nociceptor Terminals. The Journal of Neuroscience 38, 2226-2245 (2018).

48. Chen, Y., et al. The prolactin receptor long isoform regulates nociceptor sensitization and opioid-induced hyperalgesia selectively in females. Science Translational Medicine 12, eaay7550 (2020).

49. Liu, X., Bae, C., Gelman, B., Chung, J.M. \& Tang, S.-J. Mechanism and role of astrogliosis in the pathogenesis of HIV-associated pain. bioRxiv, 2021.2004.2028.441838 (2021). 
50. Prescott, S.A., Sejnowski, T.J. \& De Koninck, Y. Reduction of anion reversal potential subverts the inhibitory control of firing rate in spinal lamina I neurons: towards a biophysical basis for neuropathic pain. Mol Pain 2, 32 (2006).

51. Ahmadi, S., Lippross, S., Neuhuber, W.L. \& Zeilhofer, H.U. PGE(2) selectively blocks inhibitory glycinergic neurotransmission onto rat superficial dorsal horn neurons. Nat Neurosci 5, 34-40 (2002).

52. Moore, K.A., et al. Partial peripheral nerve injury promotes a selective loss of GABAergic inhibition in the superficial dorsal horn of the spinal cord. $J$ Neurosci 22, 6724-6731 (2002).

53. Tsuda, M., et al. JAK-STAT3 pathway regulates spinal astrocyte proliferation and neuropathic pain maintenance in rats. Brain Mar 2. [Epub ahead of print](2011).

54. Ohmichi, M., et al. Activated spinal astrocytes are involved in the maintenance of chronic widespread mechanical hyperalgesia after cast immobilization. Molecular Pain 10(2014).

55. Sasaki, M., et al. Serotonin Plays a Key Role in the Development of Opioid-Induced Hyperalgesia in Mice. The Journal of Pain (2021).

56. Sofroniew, M.V. \& Vinters, H.V. Astrocytes: biology and pathology. Acta neuropathologica 119, 7-35 (2010).

57. Kao, S.-C., et al. Absence of $\mu$ opioid receptor mRNA expression in astrocytes and microglia of rat spinal cord. NeuroReport 23, 378-384 310.1097/WNR.1090b1013e3283522e3283521b (2012).

58. Nomachi, A., et al. Receptor Tyrosine Kinase Ror2 Mediates Wnt5a-induced Polarized Cell Migration by Activating c-Jun N-terminal Kinase via Actin-binding Protein Filamin A. Journal of Biological Chemistry 283, 27973-27981 (2008).

59. Bush, T.G., et al. Fulminant Jejuno-lleitis following Ablation of Enteric Glia in Adult Transgenic Mice. Cell 93, 189 (1998).

60. Callahan, B.L., Gil, A.S., Levesque, A. \& Mogil, J.S. Modulation of mechanical and thermal nociceptive sensitivity in the laboratory mouse by behavioral state. J Pain 9(2008).

61. Bae, C., et al. Mitochondrial superoxide increases excitatory synaptic strength in spinal dorsal horn neurons of neuropathic mice. Molecular Pain 14, 1744806918797032 (2018).

62. Lee, K.Y., et al. Low-intensity, Kilohertz Frequency Spinal Cord Stimulation Differently Affects Excitatory and Inhibitory Neurons in the Rodent Superficial Dorsal Horn. Neuroscience 428, 132-139 (2020).

\section{ACKNOWLEDGEMENT}

This work was supported by NIH grants R01NS079166 (SJT), R01DA036165 (SJT), R01NS095747 (SJT) and 1R01DA050530 (SJT, JMC) and the Cecil H. and Ida M. Green Distinguished Chair in Neuroscience and Cell Biology (SJT). 


\section{FIGURES}
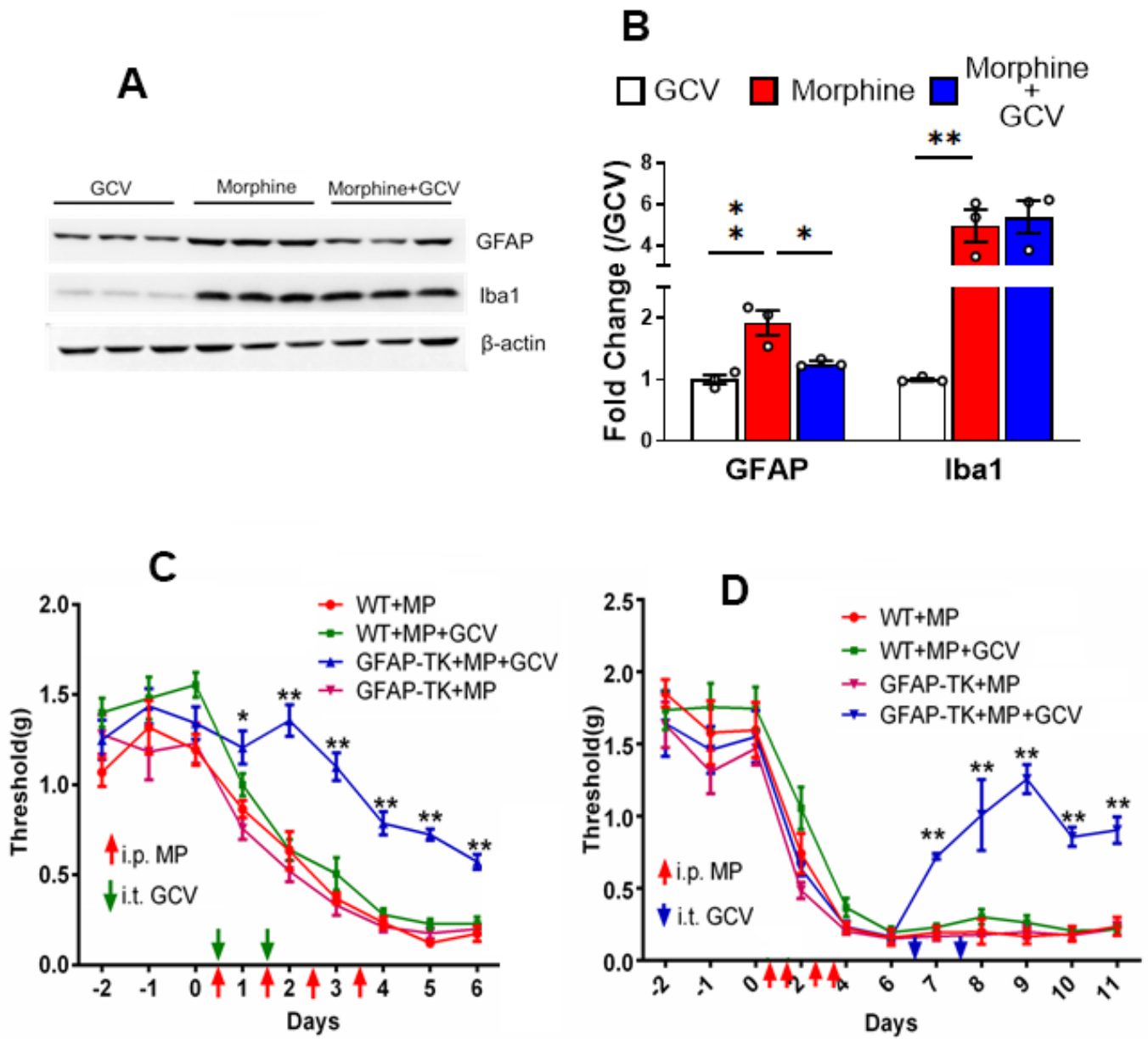

Figure 1. Reactive astrocytes are critical for morphine-induced hyperalgesia. (A) Immunoblotting analysis of GFAP and lba1 in the spinal cord of the GFAP-TK transgenic mice treated with GCV, morphine or GCV+morphine using the drug administration paradigm shown in C. Mice were euthanized for spinal cord collection at day 7. (B) Quantitative summary of band intensity of immunoblots. GCV abolished morphine-induced up-regulation of GFAP but not Iba1, indicating GCV ablated specifically reactive astrocytes but not reactive microglia in GFAP-TK mice. GFAP and Iba1 protein levels were determined by immunoblotting analysis. ${ }^{*} \mathrm{p}<0.05$; ${ }^{* *} \mathrm{p}<0.01$; ns, $p>0.05$. (C) Effect of astrogliosis ablation on OIH. GFAP-TK mice expressed mechanical OIH similarly as WT mice. Injections of GCV (green arrows; $5 \mathrm{mg} / \mathrm{kg}$; i.t.) during the early phase of morphine (MP) administration (red arrows; $20 \mathrm{mg} / \mathrm{kg}$; i.p.) inhibited the morphine-induced increase of mechanical sensitivity, measured by von Frey tests, specifically in GFAP-TK transgenic but not in WT mice ( $n=6 /$ group). (D) 
Effect of astrogliosis ablation on OIH maintenance. Injections of GCV (blue arrows; i.t.) after OIH was established by morphine administration (red arrows; i.p.) reversed the morphine-induced increase of mechanical sensitivity in GFAP-TK transgenic but not in WT mice. ( $n=6 /$ group).

A
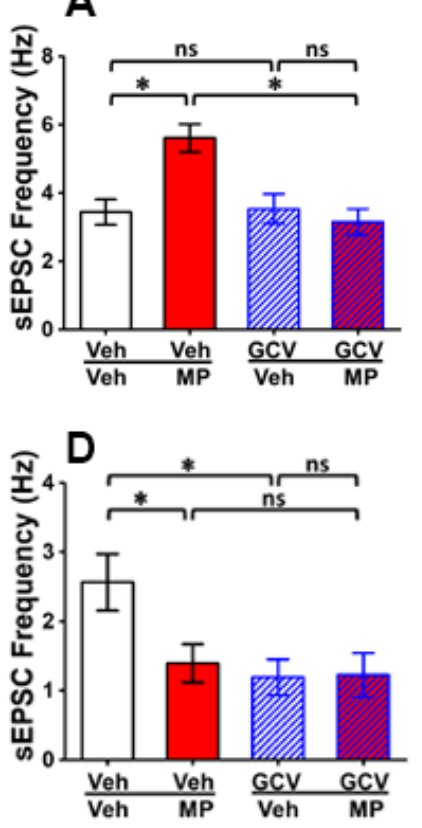

B
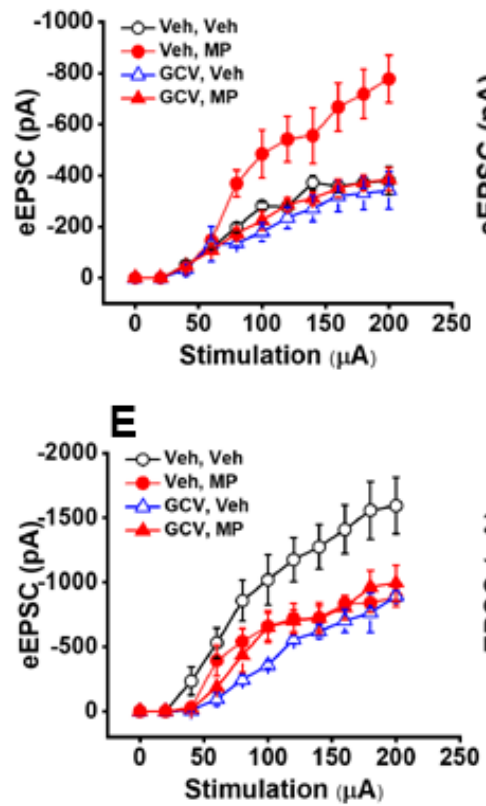
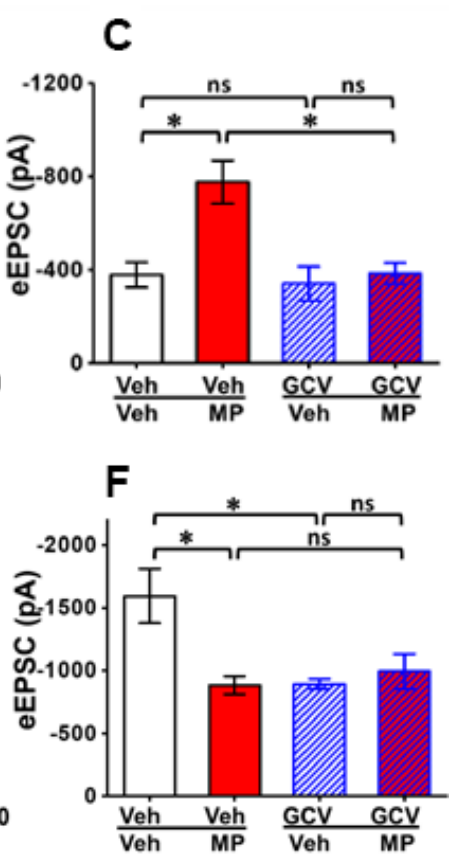

Figure 2. Reactive astrocytes are critical for morphine-induced NCP in the SDH. (A) Effect of astrocytic ablation on sEPSCs of non-tonic firing neurons in the SDH of GFAP-TK transgenic mice. Whole-cell patch recording was performed from spinal slices prepared from GFAP-TK transgenic mice (day 7) treated with morphine and/or GCV according to the paradigm shown in Fig. 1C. Morphine increased sEPSC frequency, whereas GCV abolished this increase. GCV did not affect basal sEPSC frequency. Veh/Veh: 21/3 (cells/mice); veh/MP: 60/5; GCV/Veh: 29/4; GCV/MP: 50/5. (B) Effect of astrogliosis ablation on eEPSCs of non-tonic firing neurons in the SDH of GFAP-TK transgenic mice. eEPSC amplitudes of patched SDH neurons were recorded from spinal slices prepared as in A. Morphine increased eEPSC amplitude, whereas GCV abolished this increase. GCV did not affect basal eEPSC amplitude. Veh/Veh: 24/3 (cells/mice); veh/MP: 48/4; GCV/Veh: 35/3; GCV/MP: 48/4 (C) Statistical analysis of eEPSC amplitude evoked by $200 \mu$ A stimulation shown in B. (D) Effect of astrogliosis ablation on sEPSCs of tonic firing neurons in the SDH of GFAP-TK transgenic mice. Morphine decreased sEPSC frequency. GCV alone also decreased sEPSC frequency. However, morphine did not further decrease sEPSC frequency after GCV treatment. Veh/Veh: 13/3 (cells/mice); veh/MP: 12/4; GCV/Veh: 20/5; GCV/MP: 26/5. (E) Effect of astrogliosis ablation on eEPSCs of tonic firing neurons in the SDH of GFAP-TK transgenic mice. Morphine decreased eEPSC amplitude. GCV alone also decreased eEPSC amplitude. However, morphine did not 
further decrease eEPSC amplitude after GCV treatment. Veh/Veh: 14/3 (cells/mice); veh/MP: 12/4; GCV/Veh: 20/3; GCV/MP: 26/4. (F) Statistical analysis of eEPSC amplitude evoked by $200 \mu \mathrm{A}$ stimulation shown in $\mathrm{E}$. ${ }^{*} \mathrm{p}<0.05 ;{ }^{* *} \mathrm{p}<0.01$; ns, $\mathrm{p}>0.05$.
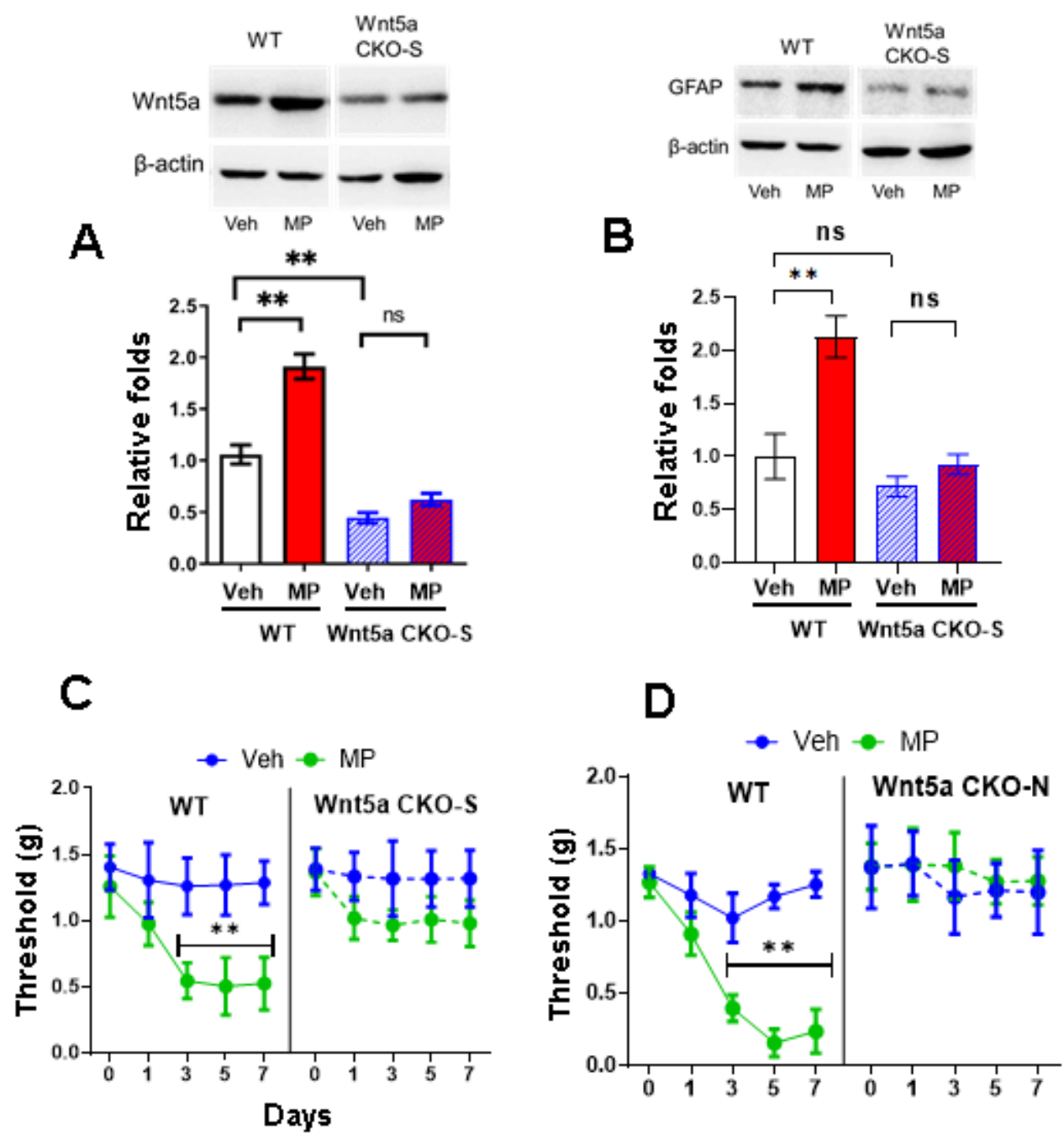

E

F
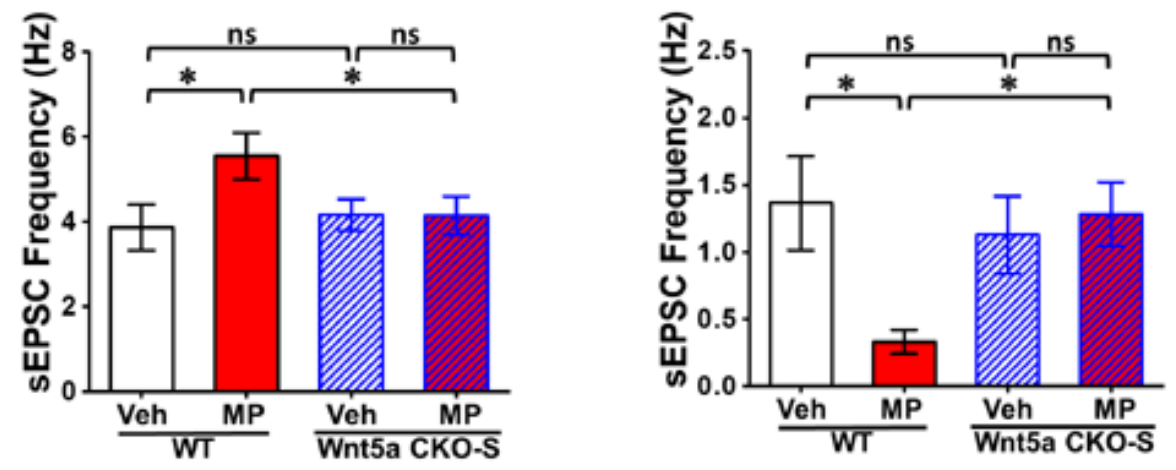

Figure 3. Neuronal Wnt5a is essential for morphine-induced astrogliosis, hyperalgesia, and NCP in the SDH. Two Wnt5a CKO mouse lines were generated, 
one by crossing floxed Wnt5a with nestin-Cre mice (Wnt5a CKO-N) and the other by crossing floxed Wnt5a with synapsin 1-Cre mice (Wnt5a CKO-S). (A) Wnt5a CKO-S (and Wnt5a CKO-N, not shown) blocked morphine (MP)-induced upregulation of Wnt5a in the spinal cord. (B) Wnt5a CKO-S (and Wnt5a CKO-N, Sup. Fig. 2) blocked morphine-induced spinal astrogliosis, as shown by GFAP upregulation. Tissues used in $A$ and $B$ were collected from mice ( $n=4 /$ group) on day 7 after morphine administration according to the paradigm shown in Fig. 1A. (C) Wnt5a CKO-S abolished OIH expression ( $n=6 /$ group). (D) Wnt5a CKO-N abolished OlH expression ( $n=6 /$ group). (E) Wnt5a CKO-S blocked the morphine-induced increase in sEPSC frequency of non-tonic firing neurons in the SDH. Veh/WT, 19/3 (cells/mice); MP/WT: 47/5; Veh/Wnt5a CKO-S, 57/5; MP/Want5a CKO-S, 33/4. (F) Wnt5a CKO-S blocked the morphine-induced decrease in SEPSC frequency of tonic firing neurons in the SDH. Veh/WT, 12/3 (cells/mice); MP/WT: 18/3; Veh/Wnt5a CKO-S, 28/5; MP/Want5a CKO-S, $13 / 4$.
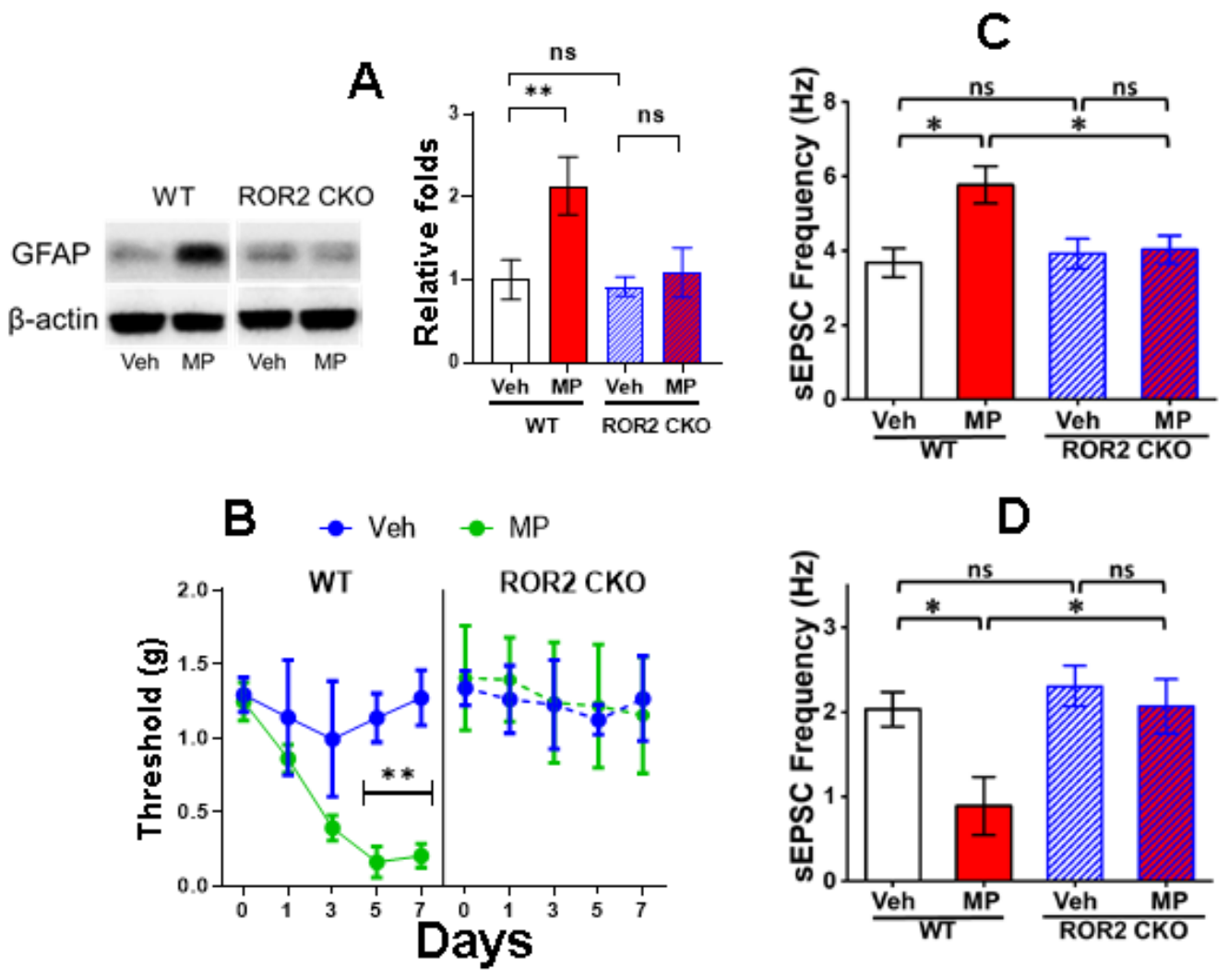

Figure 4. Astrocytic ROR2 is required for morphine-induced astrogliosis, hyperalgesia, and NCP in the SDH. (A) Astrocytic ROR2 CKO blocked morphine-induced astrogliosis. Spinal cords were collected from mice ( $n=4 / g r o u p)$ on day 7 after morphine administration according to the paradigm shown in Fig. 1A. (B) Astrocytic ROR2 CKO blocked OIH expression ( $n=6 /$ group). (C) Astrocytic ROR2 CKO blocked the morphine-induced increase in SEPSC frequency of non-tonic firing neurons in the SDH. Veh/WT, 32/3 (cells/mice); MP/WT: 53/5; Veh/ROR2 CKO, 45/3; MP/ROR2 CKO, 47/4. (D) Astrocytic ROR2 CKO blocked the morphine-induced decrease in 
SEPSC frequency of tonic firing neurons in the SDH. Veh/WT, 19/3 (cells/mice);

MP/WT: 18/3; Veh/ROR2 CKO, 22/3; MP/ROR2 CKO, 12/3.
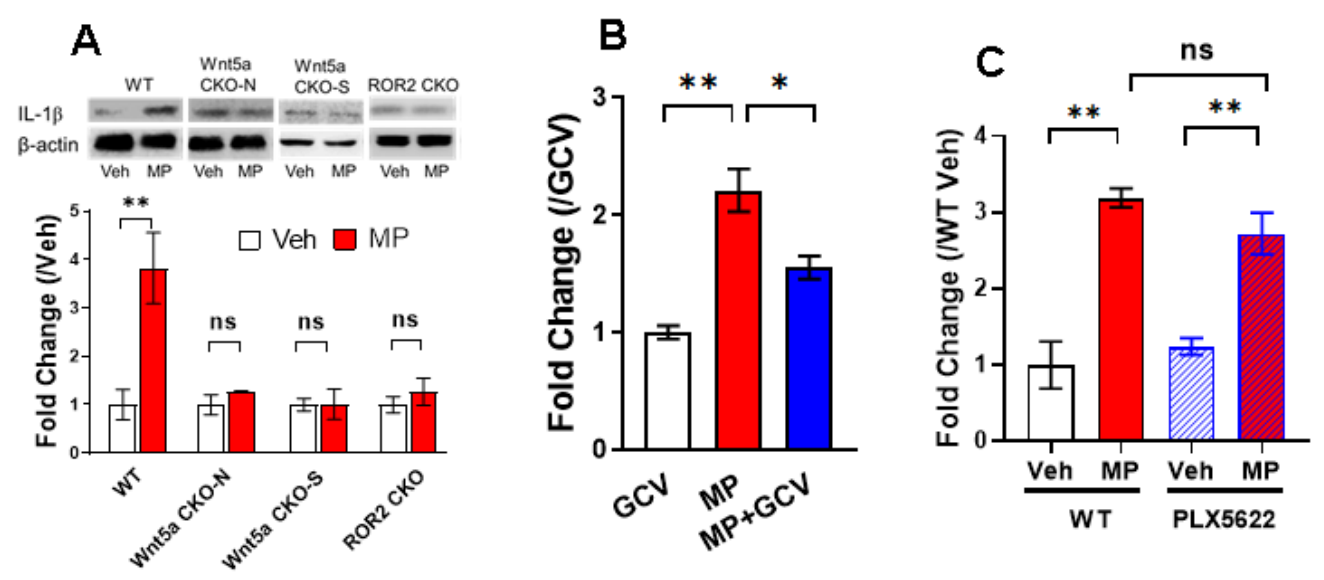

Figure 5. Morphine induces IL-1 $\beta$ activation (cleavage) via Wnt5a-ROR2 signaling-dependent astrogliosis. (A) Neuronal Wnt5a CKO and astrocytic ROR2 CKO blocked morphine-induced IL-1 $\beta$ upregulation. Spinal cords were collected for immunoblotting from mice ( $\mathrm{n}=4$ /group) on day 7 after morphine administration according to the paradigm shown in Fig. 1A. (B) Astrogliosis ablation in GFAP-TK mice inhibited morphine-induced spinal IL-1 $\beta$ upregulation as revealed by immunoblotting ( $n=4 /$ group). (C) Microglial ablation by PLX5622 did not affect morphine-induced spinal IL-1 $\beta$ upregulation as revealed by immunoblotting ( $n=4 /$ group). 

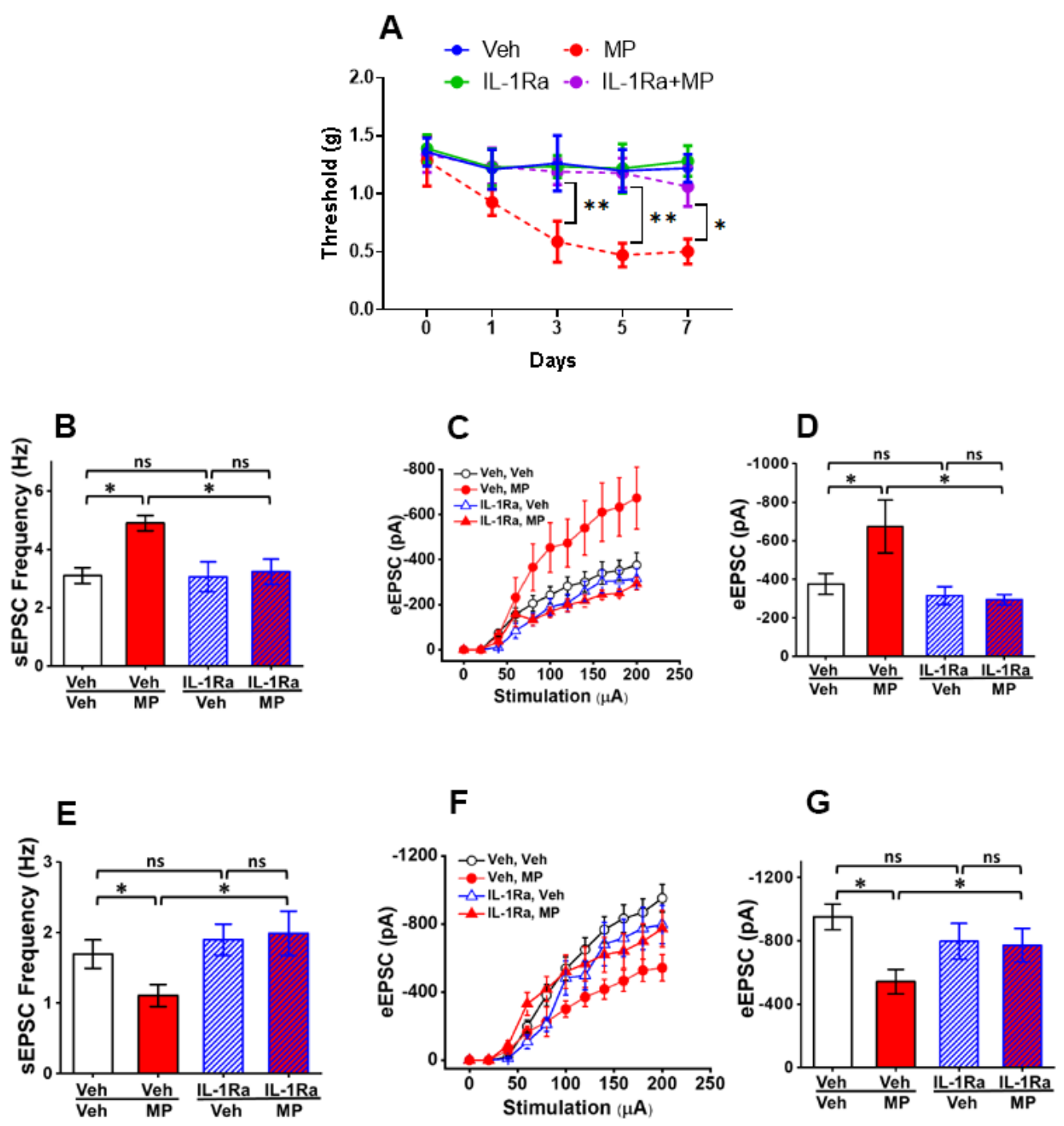

Figure 6. IL-1Ra blocks OIH and NCP. (A) The IL-1 $\beta$ receptor antagonist IL-1Ra blocked OIH expression. Morphine was administered according to the paradigm in Fig. $1 \mathrm{C}$, and IL-1Ra was administered daily on the first 3 days $(20 \mu \mathrm{g} / \mathrm{kg}$, i.t.). (B) IL-1Ra impaired the morphine-induced increase in sEPSC frequency of non-tonic firing SDH neurons (Veh/Veh: 24/3 (cells/mice); veh/MP: 33/5; IL-1Ra/Veh: 22/5; IL-1Ra/MP: $44 / 4)$ in spinal slices prepared on day 7 from mice that received drug administration as infIG. 2A. (C, D) IL-1Ra abolished the morphine-induced increase in eEPSC amplitude of non-tonic firing neurons in the $\mathrm{SDH}$; $\mathrm{D}$ shows statistical analysis of eEPSC amplitudes evoked by $200 \mu \mathrm{A}$ stimulation shown in C (Veh/Veh: 24/3 (cells/mice); veh/MP: 28/5; IL-1Ra/Veh: 19/3; IL-1Ra/MP: 40/5). (E) IL-1Ra blocked the morphine-induced decrease in sEPSC frequency of SDH inhibitory neurons from GAD67-GFP transgenic mice (Veh/Veh: 67/3 (cells/mice); veh/MP: 51/5; IL-1Ra/Veh: 35/5; IL-1Ra/MP: 44/4). (F, G) IL-1Ra abolished the morphine-induced decrease in eEPSC amplitude of SDH inhibitory neurons; G shows statistical analysis of eEPSC amplitudes evoked by $200 \mu \mathrm{A}$ stimulation shown in I (Veh/Veh: $42 / 4$ (cells/mice); 
veh/MP: 43/5; IL-1Ra/Veh: 31/3; IL-1Ra/MP: 39/4). * $\mathrm{p}<0.05 ;{ }^{* *} \mathrm{p}<0.01 ; \mathrm{ns}, \mathrm{p}>0.05$.

A

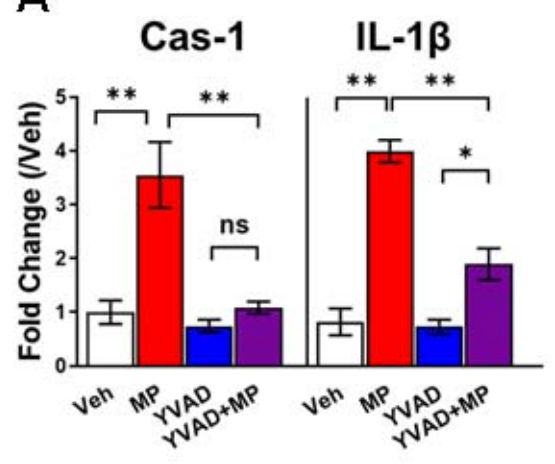

C

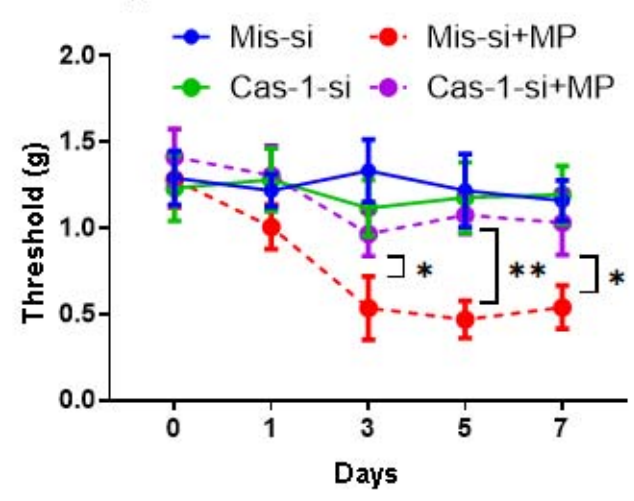

$\mathrm{B} \rightarrow \mathrm{Veh} \rightarrow \mathrm{MP}$

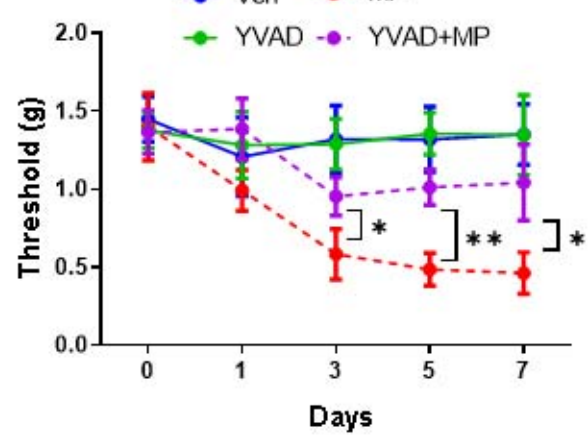

D

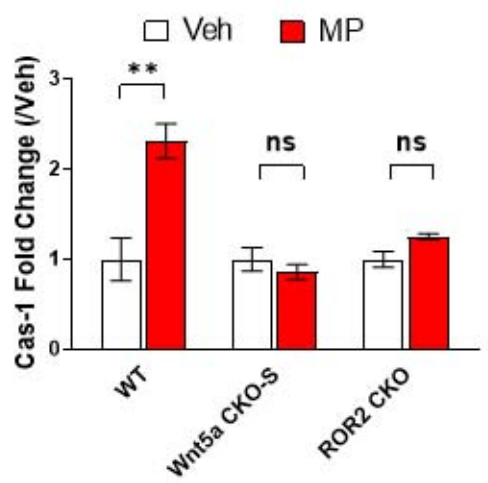

Figure 7. The Wnt5a-ROR2 signaling pathway controls inflammasome activation during $\mathrm{OIH}$ development. (A) Morphine activated the inflammasome in the spinal cord as measured by the upregulation of Cas- 1 and IL-1 $\beta$. Morphine-induced inflammasome activation was impaired by AC-YVAD-CMK (YVAD), a selective inhibitor of Cas-1. (B) YVAD impaired the expression of mechanical OIH. Morphine was administered according the paradigm shown in Fig. 1A, and YVAD was administered daily on the first 4 days ( $4 \mathrm{nmol} / \mathrm{kg}$, i.t.). (C) Cas- 1 siRNA (Cas-si) but not mismatch (silencer negative control) siRNA (Mis-si) blocked mechanical OIH expression. siRNA was administered daily on the first 4 days ( $3 \mathrm{nmol} / \mathrm{kg}$, i.t.). (D) Morphine-induced inflammasome activation, measured by Cas-1 immunoblotting, was blocked by neuronal Wnt5a-CKO-S or astrocytic ROR2 CKO. ${ }^{*} p<0.05$; ${ }^{* *} p<0.01$; ns, $\mathrm{p}>0.05$. 


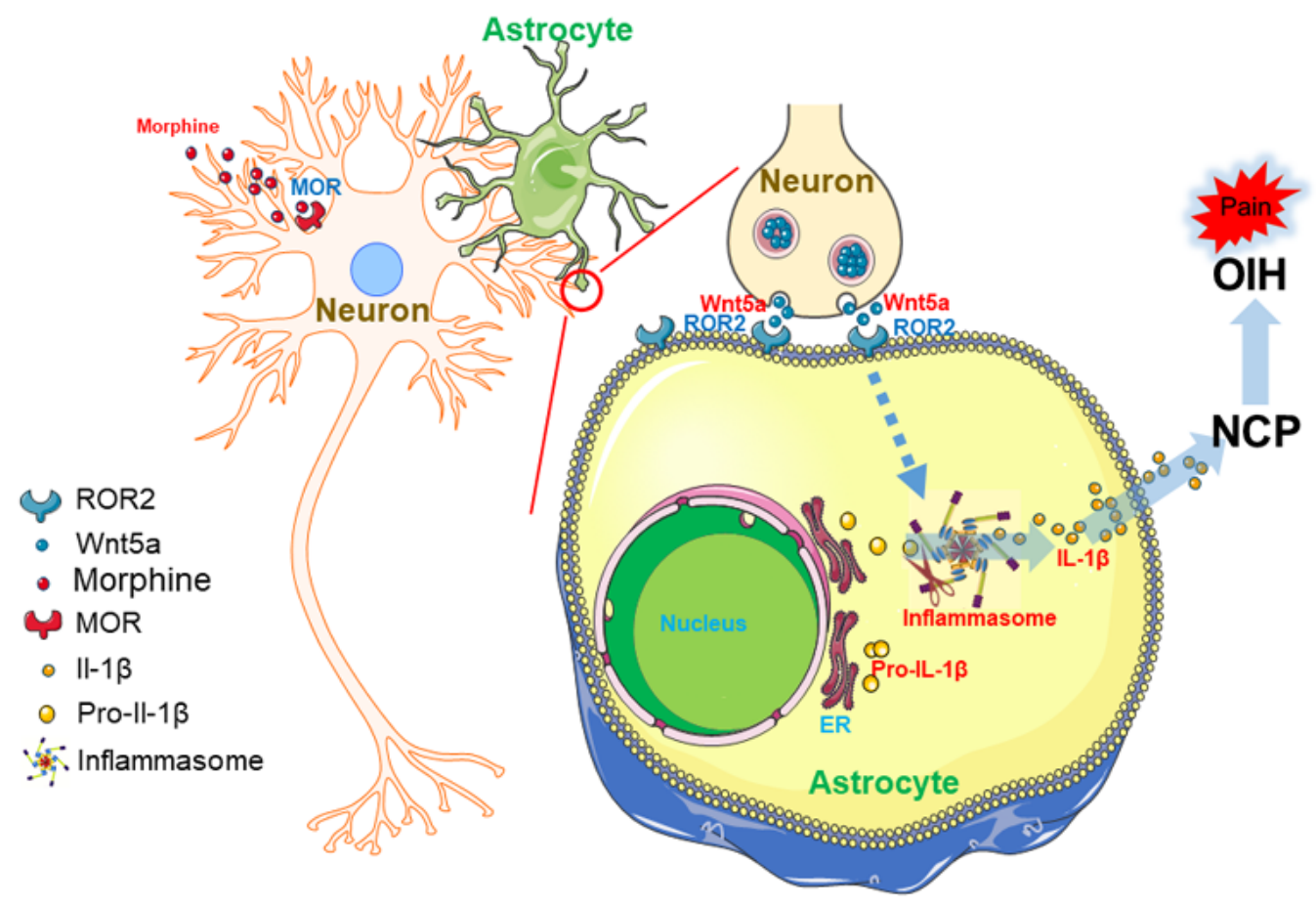

Figure 8. Model of the neuron-to-astrocyte Wnt5a-ROR2 signaling pathway in $\mathrm{OIH}$ development. Wnt5a-ROR2 signaling controls morphine-induced astrogliosis and astrocytic IL-1 $\beta$ activation. IL-1 $\beta$ is activated by the inflammasome, and feedback to neurons induces $\mathrm{OIH}$. 


\section{Materials \& Methods}

\section{Animals}

Adult male C57BL/6 mice (9-10?weeks of age) and GFAP-TK (B6.Cg-Tg(Gfap-TK)7.1Mvs/J) mice $^{59}$ were obtained from Jackson Laboratory (Bar Harbor, ME). Floxed Wnt5a mice were generated as previously described ${ }^{35}$. To delete Wnt5a in neurons, floxed Wnt5a mice were crossed with B6.Cg-Tg (Syn1-cre) 671Jxm/J mice ${ }^{36}$ (Jackson Laboratory) to generate Syn1-cre/Wnt5a flox/flox mice. To delete Wnt5a in neural stem cells, floxed Wnt5a mice were crossed with B6.Cg-Tg(Nes-cre)1K/n/J mice ${ }^{37}$ (Jackson Laboratory) to generate nestin-cre/Wnt5a flox/flox mice. To delete ROR2 in astrocytes, floxed ROR2 mice ${ }^{38}$ (Jackson Laboratory) were crossed with B6.Cg-Tg(GFAP-cre)77.6Mvs/2J mice ${ }^{39}$ (Jackson Laboratory) to generate GFAP-cre/ROR2 ${ }^{\text {flox/flox }}$ mice. Animal protocols were approved by the Institutional Animal Care and Use Committee of the University of Texas Medical Branch.

\section{Materials}

Morphine sulfate was purchased from West-Ward (Eatontown, NJ). PLX5622-containing rodent diet (1 kg containing $1200 \mathrm{mg}$ PLX5622) was purchased from Research Diets (New Brunswick, NJ). Ganciclovir sodium was purchased from Advanced ChemBlocks Inc. (Burlingame, CA). Interleukin 1 receptor antagonist (IL-1Ra) protein was purchased from R\&D Systems Inc. (Minneapolis, MN). AC-YVAD-CMK, a selective inhibitor of caspase-1, was purchased from Sigma-Aldrich (St. Louis, MO). Caspase-1 siRNA was purchased from Thermo Fisher Scientific (Waltham, MA). Antibodies used for immunoblotting were anti-IBa1 (1:1000; Wako, 016-20001); anti-GFAP (1:1000; Millipore, MAB360), anti- $\beta$-actin (1:1000; Santa Cruz Biotechnology, sc-1616-R), anti-IL-1ß (1:500; Novus Biologicals, NB600-633), anti-Wnt5a (1:1000; R\&D, MAB645), anti-caspase-1 (1:1000; Adiogen Corporation, AG-20B-0042), and anti-COX-2 (1:1000; Abcam, ab179800). Antibodies used for immunohistochemistry were anti-GFAP (1:200; Millipore, AB5541) and anti-IL-1 $(1: 200$; Novus Biologicals, NB600-633).

\section{Drug administration}

Mice were intraperitoneally injected with morphine sulfate $(20 \mathrm{mg} / \mathrm{kg})$ daily for 4 consecutive days to establish morphine-induced hyperalgesia. For microglial ablation, C57BL/6 mice were fed a rodent diet containing PLX5622 starting 5 days prior to morphine administration and continuing until the end of experimentation. To ablate reactive astrocytes, GFAP-TK or control WT mice were administered ganciclovir (5 mg/kg) by intrathecal (i.t.) injection for 2 consecutive days during the inductive or maintenance phase of opioid-induced hyperalgesia. AC-YVAD-CMK (4 nmol/ $\mathrm{kg}$ ) or siRNA (3 nmol/ $\mathrm{kg})$ were injected i.t. daily for the first 4 days. IL-1Ra $(20 \mu \mathrm{g} / \mathrm{kg})$ was injected i.t. daily for the first 3 days.

\section{Measurement of mechanical nociception by von Frey test}


Mechanical nociceptive hypersensitivity in mice was measured as previously described ${ }^{60}$. The plantar surface of the hind paw of mice in a resting state was stimulated with calibrated von Frey filaments (Stoelting, Wood Dale, IL), and paw withdrawal threshold was determined using the Dixon up and down paradigm. All tests were conducted $2 \mathrm{~h}$ prior to drug administration by an experimenter blind to the treatments received by individual animals.

\section{Western blotting analysis}

Mice were anesthetized with 3\% isoflurane and sacrificed to collect L4-L6 lumbar spinal cord segments. Tissue was homogenized in RIPA lysis buffer (1\% Nonidet P-40, 50]mM Tris-HCI $\mathrm{pH} 7.4,150$ ? $\mathrm{mM} \mathrm{NaCl}, 0.5 \%$ sodium deoxycholate, and 1] $\mathrm{mM}$ EDTA, $\mathrm{pH}$ 8.0) with a protease inhibitor cocktail (Sigma). BCA Protein Assay kits (Thermo Fisher) were used to determine protein concentrations. Equal amounts of protein $(2 \mu \mathrm{g})$ were loaded and separated by $12 \%$ SDS-PAGE and transferred to polyvinylidene fluoride membranes. The membranes were blocked in 5\% nonfat milk in Tris-buffered saline with $0.1 \%$ Tween 20 (TBST) for $1 \mathrm{~h}$ at room temperature and then incubated with primary antibodies in TBST buffer overnight at $4^{\circ} \mathrm{C}$. After washing with TBST buffer (three times for $10 \mathrm{~min}$ at room temperature), membranes were incubated with HRP-conjugated secondary antibody. Enhanced chemiluminescence kits (Pierce) were used to visualize protein bands, and NIH ImageJ software was used for quantification. $\beta$-actin was used as a loading control.

\section{Immunohistochemistry}

Mice were anesthetized with $3 \%$ isoflurane and transcardially perfused with 20 la $\mathrm{ml}$ of 0.01 $\mathrm{M}$ phosphate-buffered saline (PBS; $0.14 \mathrm{M} \mathrm{NaCl}, 0.0027 \mathrm{M} \mathrm{KCl}, 0.010 \mathrm{M} \mathrm{PO}_{4}{ }^{3}$ ) followed by 30 [? $\mathrm{ml}$ of $4 \%$ paraformaldehyde (PFA) in 0.01? $\mathrm{M}$ PBS. L4 and L5 lumbar spinal cord segments were fixed in $4 \%$ PFA solution for 12 ?h at $4^{\circ} \mathrm{C}$, dehydrated with $30 \%$ sucrose solution in PBS for $24 \mathrm{~h}$ at $4^{\circ} \mathrm{C}$, and embedded in optimal cutting temperature medium (Tissue-Tek). Tissue was sectioned (15 $\mu \mathrm{m}$ ) on a cryostat (Leica CM 1900) and mounted onto Superfrost Plus microscope slides. Sections were incubated in blocking solution containing $5 \%$ bovine serum albumin (BSA) and $0.3 \%$ Triton X-100 in 0.01? $\mathrm{M}$ PBS for 1? $\mathrm{h}$ at room temperature and then in chicken anti-GFAP (1:200; Millipore, AB5541) or rabbit IL-1 $\beta$ (1:200; Novus Biologicals, NB600-633) in blocking solution overnight. Sections were washed with $0.01 \mathrm{M}$ PBS (three times for $10 \mathrm{~min}$ at room temperature) and incubated with FITC- or Texas Red-conjugated secondary antibody (1:200; Jackson ImmunoResearch Laboratories) in dilution buffer (1\% BSA and $0.3 \%$ Triton X-100 in 0.01 M PBS) before mounting. Finally, sections were stained with DAPI (Sigma) to visualize nuclei. Animal species-matched IgG was used as a negative control for primary antibodies. Images were collected on a confocal microscope (model A1, Nikon s).

\section{Patch-clamp recording of dorsal horn neurons in ex vivo spinal cord slices}


Spinal cord slices were prepared as previously described ${ }^{61}$. Briefly, the spinal cord was sliced transversely at a thickness of $350 \mu \mathrm{m}$ using a vibratome (Leica VT1200S, Buffalo Grove, IL) in cold $\left(4^{\circ} \mathrm{C}\right.$ ) N-methyl-D-glucamine (NMDG) solution (93 mM NMDG, $2.5 \mathrm{mM} \mathrm{KCl}, 1.2 \mathrm{mM}$ $\mathrm{NaH}_{2} \mathrm{PO}_{4}, 30 \mathrm{mM} \mathrm{NaHCO}$, $20 \mathrm{mM}$ HEPES, $25 \mathrm{mM}$ glucose, $5 \mathrm{mM}$ sodium ascorbate, $2 \mathrm{mM}$ thiourea, $3 \mathrm{mM}$ sodium pyruvate, $10 \mathrm{mM} \mathrm{MgSO}_{4}$, and $0.5 \mathrm{mM} \mathrm{CaCl}_{2}, \mathrm{pH} 7.4$ ) saturated with $95 \% \mathrm{O}_{2}$ and $5 \% \mathrm{CO}_{2}$. Whole-cell recordings were performed from randomly selected neurons in lamina II in artificial cerebrospinal fluid (ACSF; $124 \mathrm{mM} \mathrm{NaCl}, 2.5 \mathrm{mM} \mathrm{KCl}, 1.2 \mathrm{mM}$

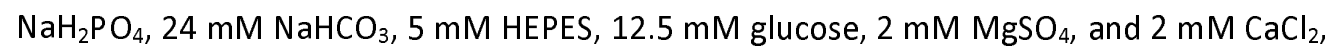
pH 7.4) using a Multiclamp 700B amplifier, DigiDATA, and pClamp software (version 10.6. Molecular Device, Sunnyvale, $\mathrm{CA}$ ) with a $10-\mathrm{kHz}$ sampling rate and $2-\mathrm{kHz}$ filtering rate. Patch pipettes (4-8 M $\Omega$ ) were filled with internal solution (120 mM K-gluconate, $10 \mathrm{mM} \mathrm{KCl}, 2 \mathrm{mM}$ Mg-ATP, $0.5 \mathrm{mM}$ Na-GTP, $0.5 \mathrm{mM}$ EGTA, $20 \mathrm{mM}$ HEPES, and $10 \mathrm{mM}$ phosphocreatine, pH 7.3). After achieving a whole-cell recording configuration, spontaneous excitatory postsynaptic currents (sEPSCs) were recorded for $60 \mathrm{~s}$ at $-65 \mathrm{mV}$ in ACSF. Evoked EPSCS (eEPSCs) were elicited by focal electrical stimulation in the vicinity of recorded neurons with a metal bipolar electrode (MicroProbes, Gaithersburg, MD). Test pulses were delivered for $0.5 \mathrm{~ms}$ at 5-s intervals, with stimulation intensities ranging from $20-200 \mu \mathrm{A}(20-\mu \mathrm{A}$ steps). Recordings were performed only when eEPSCS were monosynaptic, based on characteristic waveforms with short latency, a single peak, and stable responses to repeated stimuli.

Recordings showing polysynaptic responses were disregarded. Neurons were characterized by their action potential firing pattern upon depolarizing current injections in current clamp mode ${ }^{62}$.

\section{Statistical analysis}

Statistical analysis was conducted with Prism 5 (GraphPad) software. Data are shown as mean? + ?]standard error of the mean (SEM). One-way ANOVA was used for immunoblotting data, and two-way ANOVA with Bonferroni post hoc tests were used for pain behavior data. Electrophysiological data are expressed as mean? + ? cells and $\mathrm{N}$ indicating the number of animals. SEPSC frequency and eEPSC amplitude were analyzed offline using Clampfit software (version 11, Molecular Devices, CA). SEPSC events were detected using the template event detection method. Electrophysiological data were analyzed using two-way ANOVA followed by Holm-Sidak multiple comparison tests. For all tests, $\mathrm{p}$ ?? $<0.05$ was considered statistically significant. Origin (version PRO 2020, OriginLab, Northampton, MA) was used to analyze data.

\section{References}

1. Corder, G., et al. Loss of $\mu$ opioid receptor signaling in nociceptors, but not microglia, abrogates morphine tolerance without disrupting analgesia. Nature Medicine 23, 164 (2017).

2. Mao, J., Price, D.D. \& Mayer, D.J. Thermal hyperalgesia in association with the development of morphine tolerance in rats: roles of excitatory amino acid receptors and protein kinase C. J Neurosci 14, 2301-2312 (1994).

3. Angst, Martin S. \& Clark, J D. Opioid-induced Hyperalgesia: A Qualitative Systematic Review. 
Anesthesiology 104, 570-587 (2006).

4. Chu, L.F., Angst, M.S. \& Clark, D. Opioid-induced Hyperalgesia in Humans: Molecular Mechanisms and Clinical Considerations. The Clinical Journal of Pain 24, 479-496 410.1097/AJP.1090b1013e31816b31812f31843 (2008).

5. Servick, K. Primed for pain. Science 354, 569-571 (2016).

6. Miller, G. Pot and pain. Science 354, 566-568 (2016).

7. Roeckel, L.-A., Le Coz, G.-M., Gavériaux-Ruff, C. \& Simonin, F. Opioid-induced hyperalgesia: Cellular and molecular mechanisms. Neuroscience 338, 160-182 (2016).

8. Drdla, R., Gassner, M., Gingl, E. \& Sandkühler, J. Induction of Synaptic Long-Term Potentiation After Opioid Withdrawal. Science 325, 207-210 (2009).

9. Ferrini, F., et al. Morphine hyperalgesia gated through microglia-mediated disruption of neuronal Cl- homeostasis. Nature Neuroscience 16, 183 (2013).

10. Liu, X., Liu, B.-L., Yang, Q., Zhou, X. \& Tang, S.-J. Microglial ablation does not affect opioid-induced hyperalgesia in rodents. bioRxiv, 2021.2004 .2027 .441650 (2021).

11. Berta, T., Liu, Y.C., Xu, Z.Z. \& Ji, R.R. Tissue plasminogen activator contributes to morphine tolerance and induces mechanical allodynia via astrocytic IL-1 $\beta$ and ERK signaling in the spinal cord of mice. Neuroscience 247, 376-385 (2013).

12. Sanna, M.D., Ghelardini, C. \& Galeotti, N. Activation of JNK pathway in spinal astrocytes contributes to acute ultra-low-dose morphine thermal hyperalgesia. Pain 156, 1265-1275 (2015).

13. Perea, G., Navarrete, M. \& Araque, A. Tripartite synapses: astrocytes process and control synaptic information. Trends in neurosciences 32, 421-431 (2009).

14. Ji, R.-R., Donnelly, C.R. \& Nedergaard, M. Astrocytes in chronic pain and itch. Nature reviews. Neuroscience 20, 667-685 (2019).

15. Barres, B.A. The mystery and magic of glia: a perspective on their roles in health and disease. Neuron 60, 430-440 (2008).

16. Khakh, B.S. \& Sofroniew, M.V. Diversity of astrocyte functions and phenotypes in neural circuits. Nature neuroscience 18, 942-952 (2015).

17. Allen, N.J. \& Eroglu, C. Cell Biology of Astrocyte-Synapse Interactions. Neuron 96, 697-708 (2017).

18. Lines, J., Martin, E.D., Kofuji, P., Aguilar, J. \& Araque, A. Astrocytes modulate sensory-evoked neuronal network activity. Nat Commun 11, 3689 (2020).

19. Li, X., Angst, M.S. \& Clark, J.D. A murine model of opioid-induced hyperalgesia. Brain research. Molecular brain research 86, 56-62 (2001).

20. Song, P. \& Zhao, Z.Q. The involvement of glial cells in the development of morphine tolerance. Neuroscience research 39, 281-286 (2001).

21. Hutchinson, M.R., et al. Possible involvement of toll-like receptor 4 /myeloid differentiation factor-2 activity of opioid inactive isomers causes spinal proinflammation and related behavioral consequences. Neuroscience 167, 880-893 (2010).

22. Bush, T.G., et al. Leukocyte infiltration, neuronal degeneration, and neurite outgrowth after ablation of scar-forming, reactive astrocytes in adult transgenic mice. Neuron 23, 297-308 (1999).

23. Punnakkal, P., von Schoultz, C., Haenraets, K., Wildner, H. \& Zeilhofer, H.U. Morphological, biophysical and synaptic properties of glutamatergic neurons of the mouse spinal dorsal horn. 
The Journal of Physiology 592, 759-776 (2014).

24. Todd, A.J. Identifying functional populations among the interneurons in laminae I-III of the spinal dorsal horn. Molecular Pain 13, 1744806917693003 (2017).

25. Yuan, S., Shi, Y. \& Tang, S.J. Wnt Signaling in the Pathogenesis of Multiple Sclerosis-Associated Chronic Pain. J Neuroimmune Pharmacology [Epub ahead of print] DOI:

10.1007/s11481-012-9370-3(2012).

26. Yuan, S., et al. A Wnt5a signaling pathway in the pathogenesis of HIV-1 gp120-induced pain. Pain 156, 1311-1319 (2015).

27. Simonetti, M. \& Kuner, R. Spinal Wnt5a Plays a Key Role in Spinal Dendritic Spine Remodeling in Neuropathic and Inflammatory Pain Models and in the Proalgesic Effects of Peripheral Wnt3a. J Neurosci 40, 6664-6677 (2020).

28. Li, B., et al. Wingless-type Mammary Tumor Virus Integration Site Family, Member 5A (Wnt5a) Regulates Human Immunodeficiency Virus Type 1 (HIV-1) Envelope Glycoprotein 120 (gp120)-induced Expression of Pro-Inflammatory Cytokines via the Ca2+/Calmodulin-dependent Protein Kinase II (CaMKII) and c-Jun N-terminal Kinase (JNK) Signaling Pathways. Journal of Biological Chemistry 288, 13610-13619 (2013).

29. Shi, Y., et al. Regulation of Wnt signaling by nociceptive in put in animal models. Molecular Pain 8, 47 (2012).

30. $\mathrm{Li}, \mathrm{Y}$. , et al. NMDA receptor activation stimulates transcription-independent rapid wnt5a protein synthesis via the MAPK signaling pathway. Molecular Brain 5, 1 (2012).

31. Chen, J., Park, C.S. \& Tang, S.J. Activity-dependent synaptic Wnt release regulates hippocampal long term potentiation. J Biol Chem 281, 11910-11916 (2006).

32. Shi, Y., Yuan, S. \& Tang, S.-J. Morphine and HIV-1 gp120 cooperatively promote pathogenesis in the spinal pain neural circuit. Molecular Pain 15, 1744806919868380 (2019).

33. Yuan, S., Shi, Y., Guo, K. \& Tang, S.-J. Nucleoside Reverse Transcriptase Inhibitors (NRTIs) Induce Pathological Pain through Wnt5a-Mediated Neuroinflammation in Aging Mice. Journal of Neuroimmune Pharmacology (2018).

34. Zhuang, Z.-Y., et al. A Peptide c-Jun N-Terminal Kinase (JNK) Inhibitor Blocks Mechanical Allodynia after Spinal Nerve Ligation: Respective Roles of JNK Activation in Primary Sensory Neurons and Spinal Astrocytes for Neuropathic Pain Development and Maintenance. The Journal of Neuroscience 26, 3551-3560 (2006).

35. Miyoshi, H., Ajima, R., Luo, C.T., Yamaguchi, T.P. \& Stappenbeck, T.S. Wnt5a potentiates TGF- $\beta$ signaling to promote colonic crypt regeneration after tissue injury. Science $\mathbf{3 3 8}, 108-113$ (2012).

36. Zhu, Y., et al. Ablation of NF1 function in neurons induces abnormal development of cerebral cortex and reactive gliosis in the brain. Genes \& Development 15, 859-876 (2001).

37. Tronche, F., et al. Disruption of the glucocorticoid receptor gene in the nervous system results in reduced anxiety. Nat Genet 23, 99 (1999).

38. Ho, H.-Y.H., et al. Wnt5a-Ror-Dishevelled signaling constitutes a core developmental pathway that controls tissue morphogenesis. Proceedings of the National Academy of Sciences 109, 4044-4051 (2012).

39. Garcia, A.D.R., Doan, N.B., Imura, T., Bush, T.G. \& Sofroniew, M.V. GFAP-expressing progenitors are the principal source of constitutive neurogenesis in adult mouse forebrain. Nat Neurosci 7, 1233 (2004). 
40. Johnston, I.N., et al. A role for proinflammatory cytokines and fractalkine in analgesia, tolerance, and subsequent pain facilitation induced by chronic intrathecal morphine. $J$ Neurosci 24, 7353-7365 (2004).

41. Hilla, A.M., Diekmann, H. \& Fischer, D. Microglia Are Irrelevant for Neuronal Degeneration and Axon Regeneration after Acute Injury. The Journal of Neuroscience 37, 6113-6124 (2017).

42. Janova, H., et al. Microglia ablation alleviates myelin-associated catatonic signs in mice. The Journal of Clinical Investigation 128(2017).

43. Reshef, R., et al. The role of microglia and their CX3CR1 signaling in adult neurogenesis in the olfactory bulb. elife 6, e30809 (2017).

44. Oliva, A.A., Jiang, M., Lam, T., Smith, K.L. \& Swann, J.W. Novel Hippocampal Interneuronal Subtypes Identified Using Transgenic Mice That Express Green Fluorescent Protein in GABAergic Interneurons. The Journal of Neuroscience 20, 3354-3368 (2000).

45. Schroder, K. \& Tschopp, J. The Inflammasomes. Cell 140, 821-832 (2010).

46. Grace, P.M., et al. Morphine paradoxically prolongs neuropathic pain in rats by amplifying spinal NLRP3 inflammasome activation. Proceedings of the National Academy of Sciences 113, E3441-E3450 (2016).

47. Araldi, D., Khomula, E.V., Ferrari, L.F. \& Levine, J.D. Fentanyl Induces Rapid Onset Hyperalgesic Priming: Type I at Peripheral and Type II at Central Nociceptor Terminals. The Journal of Neuroscience 38, 2226-2245 (2018).

48. Chen, Y., et al. The prolactin receptor long isoform regulates nociceptor sensitization and opioid-induced hyperalgesia selectively in females. Science Translational Medicine 12, eaay7550 (2020).

49. Liu, X., Bae, C., Gelman, B., Chung, J.M. \& Tang, S.-J. Mechanism and role of astrogliosis in the pathogenesis of HIV-associated pain. bioRxiv, 2021.2004.2028.441838 (2021).

50. Prescott, S.A., Sejnowski, T.J. \& De Koninck, Y. Reduction of anion reversal potential subverts the inhibitory control of firing rate in spinal lamina I neurons: towards a biophysical basis for neuropathic pain. Mol Pain 2, 32 (2006).

51. Ahmadi, S., Lippross, S., Neuhuber, W.L. \& Zeilhofer, H.U. PGE(2) selectively blocks inhibitory glycinergic neurotransmission onto rat superficial dorsal horn neurons. Nat Neurosci 5, 34-40 (2002).

52. Moore, K.A., et al. Partial peripheral nerve injury promotes a selective loss of GABAergic inhibition in the superficial dorsal horn of the spinal cord. J Neurosci 22, 6724-6731 (2002).

53. Tsuda, M., et al. JAK-STAT3 pathway regulates spinal astrocyte proliferation and neuropathic pain maintenance in rats. Brain Mar 2. [Epub ahead of print](2011).

54. Ohmichi, M., et al. Activated spinal astrocytes are involved in the maintenance of chronic widespread mechanical hyperalgesia after cast immobilization. Molecular Pain 10(2014).

55. Sasaki, M., et al. Serotonin Plays a Key Role in the Development of Opioid-Induced Hyperalgesia in Mice. The Journal of Pain (2021).

56. Sofroniew, M.V. \& Vinters, H.V. Astrocytes: biology and pathology. Acta neuropathologica 119, 7-35 (2010).

57. Kao, S.-C., et al. Absence of $\mu$ opioid receptor mRNA expression in astrocytes and microglia of rat spinal cord. NeuroReport 23, 378-384 310.1097/WNR.1090b1013e3283522e3283521b (2012).

58. Nomachi, A., et al. Receptor Tyrosine Kinase Ror2 Mediates Wnt5a-induced Polarized Cell 
Migration by Activating c-Jun N-terminal Kinase via Actin-binding Protein Filamin A. Journal of Biological Chemistry 283, 27973-27981 (2008).

59. Bush, T.G., et al. Fulminant Jejuno-lleitis following Ablation of Enteric Glia in Adult Transgenic Mice. Cell 93, 189 (1998).

60. Callahan, B.L., Gil, A.S., Levesque, A. \& Mogil, J.S. Modulation of mechanical and thermal

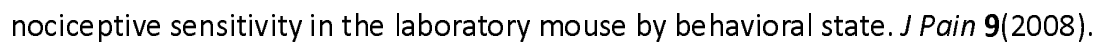

61. Bae, C., et al. Mitochondrial superoxide increases excitatory synaptic strength in spinal dorsal horn neurons of neuropathic mice. Molecular Pain 14, 1744806918797032 (2018).

62. Lee, K.Y., et al. Low-intensity, Kilohertz Frequency Spinal Cord Stimulation Differently Affects Excitatory and Inhibitory Neurons in the Rodent Superficial Dorsal Horn. Neuroscience 428, 132-139 (2020).

\section{Supplemental Figures}

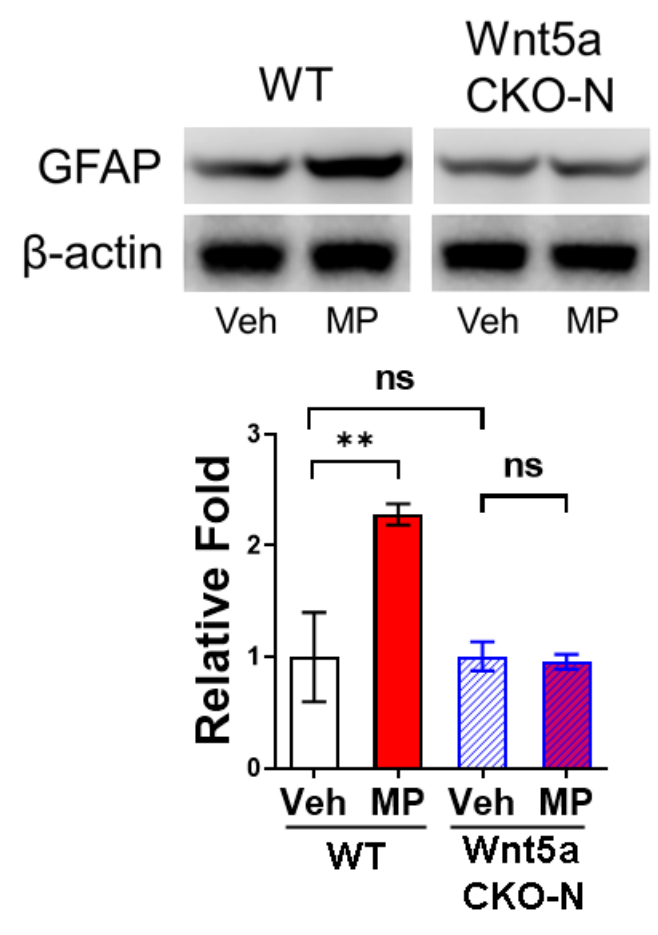

Supplemental Figure 1. Wnt5a CKO-N blocked morphine-induced astrogliosis in the spinal cord. 